\title{
Motivic Analogues of MO and MSO
}

\author{
by \\ Dondi Ellis \\ A dissertation submitted in partial fulfillment \\ of the requirements for the degree of \\ Doctor of Philosophy \\ (Mathematics) \\ in The University of Michigan \\ 2017
}

Doctoral Committee:

Professor Igor Kriz, Chair

Professor Bhargav Bhatt

Professor James T. Liu

Professor Peter Scott

Professor Karen E. Smith 


\section{Dondi Ellis \\ dondi@umich.edu \\ ORCID iD: 0000-0001-5070-2666}

(C) Dondi Ellis 2107 
To my parents and my beautiful wife Nana. 


\section{ACKNOWLEDGEMENTS}

There are many people who were essential to the production of this thesis. First and foremost I wish to thank my advisor, Igor Kriz, who has been a continuous source of motivation and inspiration throughout my graduate studies. I would also like to thank the rest of my thesis committee: Bhargav Bhatt, James Liu, Peter Scott, and Karen Smith.

I could not have produced this work without the support, encouragement, patience, and unwavering love of my wife, Nana. Her tolerance of my occasional illtempered moods is a testament in itself of her unyielding devotion and love. I thank my parents, Dondi and Donita, for their faith in me and for allowing me to be as ambitious as I wanted. It was under their watchful eye that I gained the drive and the ability to accomplish so much.

Finally, I would like to thank my grandmother Colleen, as well as the rest of my family, for all of their love and support over the years. 


\section{TABLE OF CONTENTS}

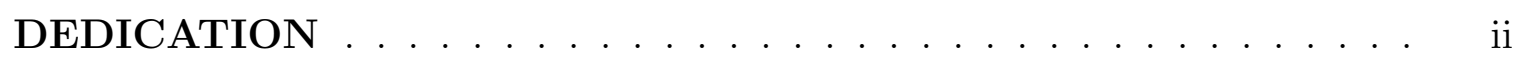

ACKNOWLEDGEMENTS ................... iii

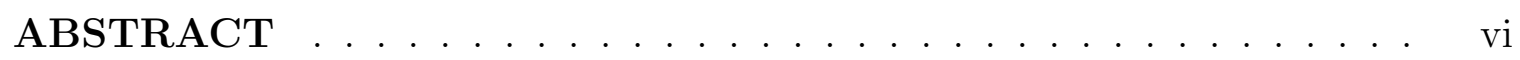

\section{CHAPTER}

I. Introduction $\ldots \ldots \ldots \ldots \ldots \ldots \ldots \ldots$

1.1 Motivic homotopy theory . . . . . . . . . . . . . . 2

1.1.1 The bigraded family of spheres . . . . . . . . . . 4

1.1.2 The stable motivic homotopy category . . . . . . 4

1.2 G-equivariant motivic homotopy theory . . . . . . . . . 5

1.2.1 The family of $C_{2}$ spheres $\ldots \ldots \ldots \ldots$

1.2.2 Two kinds of classifying spaces . . . . . . . . . 6

$1.3 G$-equivariant stable motivic homotopy theory . . . . . . . 7

II. A motivic analogue of $\mathrm{MO} \ldots \ldots \ldots \ldots$

2.1 The construction of MGLO $\ldots \ldots \ldots \ldots \ldots$

2.1 .1 Quadratic forms . . . . . . . . . . . . . . . . 12

2.1 .2 Cellularity . . . . . . . . . . . . . . . 17

2.1.3 Two-sided bar construction . . . . . . . . . . . . 19

2.1.4 The prespectrum for MGLO . . . . . . . . . . . 20

2.2 Computing the coefficients of MGLO . . . . . . . . . . 22

2.2.1 Dual Motivic Steenrod Algebra . . . . . . . . . . . 24

$2.2 .22=\eta=0$ in $\mathrm{MGLO}_{\star} \ldots \ldots \ldots \ldots \ldots \ldots$

2.2 .3 Comodule structure of MGLO . . . . . . . . . 26

2.2.4 Applying the Motivic Hurewicz Theorem . . . . . . 27

2.2.5 $\mathrm{MGLO}_{\star}$ and a comparison with $\mathrm{MO}_{*} \ldots \ldots \ldots$

2.2.6 The topological realization of MGLO over $\mathbb{R} \ldots$. . . 29

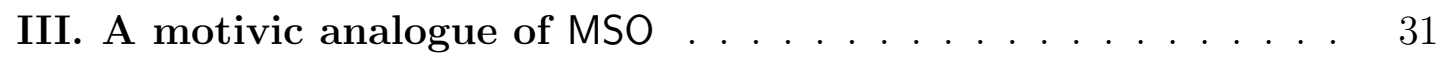


3.1 Computing the coefficients of MSLO . . . . . . . . . 31

3.1.1 Calculating the $\mathbb{Z} / 2$ cohomology of MSLO . . . . . 34

3.1.2 $H_{\star}(H \mathbb{Z})$ comodule structure of MSLO . . . . . . . 36

3.1.3 Calculating the $\mathbb{Z} / p$ cohomology of MSLO for $p$ an odd prime ................ 36

3.1.4 Calculating the coefficients of $\mathrm{MSLO}_{p}$ for $p$ an odd prime . . . . . . . . . . . . 37

3.1.5 $H \mathbb{Z} / 2_{\star}$-algebra structure of $H_{\star}(H \mathbb{Z} ; \mathbb{Z} / 2) \ldots \ldots . .40$

3.1.6 The $\mathrm{Sq}^{1}$ cohomology . . . . . . . . . . . . . 42

3.1.7 A motivic version of Wall's Theorem . . . . . . . . . 45

3.1 .8 The homotopy type of MSLO . . . . . . . . . . 50

3.1.9 The dimension of the $H \mathbb{Z} / 2$ suspensions . . . . . . . 50

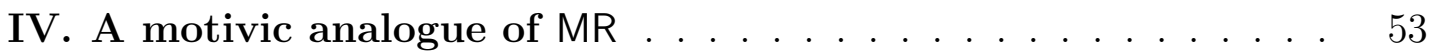

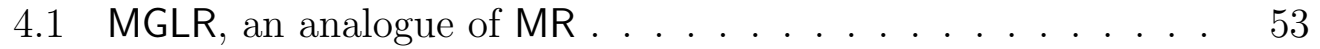

4.1.1 The $\lambda$ twist . . . . . . . . . . . . 54

4.2 Calculating the coefficients of $\theta^{-1} \lambda^{-1}$ MGLR $\ldots \ldots \ldots 58$

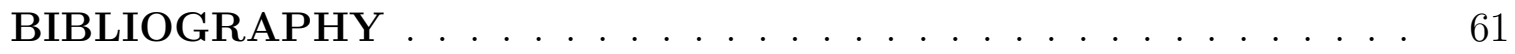




\title{
ABSTRACT \\ Motivic Analogues of MO and MSO \\ by \\ Dondi Ellis
}

Chair: Igor Kriz

\begin{abstract}
This thesis makes progress in computing the coefficients of Algebraic Hermitian Cobordism (MGLR), a motivic $C_{2}$-equivariant spectrum constructed by P. $\mathrm{Hu}$, I. Kriz, and K. Ormsby. In the process of my research, I realized it would be possible to construct motivic analogues of unoriented and oriented cobordism, which I refer to as MGLO and MSLO respectively. In chapters 2-3 of my thesis, I construct MGLO and MLSO and give a concrete description of the homotopy groups of each of them. In particular, my work on MGLO gives an answer to a question of Jack Morava. Using the tools of Tate cohomology and my computation of the coefficients of MGLO, the thesis ends with a computation of a localization of the homotopy groups of MGLR.
\end{abstract}




\section{CHAPTER I}

\section{Introduction}

My thesis is an extension of the work of P. Hu, I. Kriz, and K. Ormsby [HKO11]. In $[\mathrm{HKO} 11]$, the authors construct a $C_{2}$-equivariant $\mathrm{E}_{\infty}$-ring spectrum MGLR. This is the algebraic version of Landweber's topological real cobordism MR [Lan67, Lan68]. Recall that MR is a $C_{2}$-equivariant analogue of complex cobordism MU. By taking the geometric fixed points of MR (i.e. $\Phi^{C_{2}}(\mathrm{MR})$ ) one obtains the unoriented cobordism ring MO of Milnor and Thom. Motivically there is an étale geometric fixed points functor $\Phi_{e t}^{C_{2}}$ satisfying $\Phi_{e t}^{C_{2}}(M G L R)=$ MGLO. The topological realization of MGLO over $\mathrm{k}=\mathbb{C}$ is $\mathrm{MO}$, and so MGLO should be thought of as a motivic analogue of MO. We will describe the cobordism spectrum MGLO fully in this thesis for $k$ any field of characteristic 0 . The answer is very beautiful, and the proofs bear great similarity to the classical case. My construction and computation of the unoriented cobordism spectrum MGLO answer a question of Jack Morava.

After having completed my work on MGLO, it became clear to me that I could construct a motivic analogue of unoriented cobordism MSO. I call this MSLO, and its construction follows the construction of its topological counterpart MSO. The key observation is that the determinant function is algebraic, and therefore the generalized orthogonal groups $O_{n}$ used to construct MGLO can be used to define special orthogonal groups $S O_{n}$. After restricting to a ground field k of characteristic 0 for 
which -1 is a square in $\mathrm{k}$, and completing at $p$ an odd prime, MSLO splits as a wedge sum of suspensions of BPGL, the motivic Brown-Peterson spectrum. After restricting to a ground field $\mathrm{k}$ of characteristic 0 for which -1 is a square in $\mathrm{k}$, and completing at the prime $p=2$, MSLO splits as a wedge sum of suspensions of motivic $H \mathbb{Z}$ and $H \mathbb{Z} / 2$.

In chapter 4, using computations relating to MGLO coupled with the tools of $C_{2}$-equivariant homotopy theory, I give a computation of the motivic $C_{2}$-equivariant spectrum MGLR (completed at 2) after inverting a twist $\lambda$ of degree $1-\sigma+\sigma \alpha-\alpha$ and a twist $\theta$ of degree $1-\alpha$. Chapters 2 and 3 pertain to MGLO and MSLO respectively. The remainder of the current chapter will serve as a reference as well as a means to establishing notation for the material which will follow. We divide Chapter 1 into two parts. Part 1 will give the non-equivariant story and Part 2 will give the equivariant story.

\subsection{Motivic homotopy theory}

Informally, Motivic homotopy theory is an answer to the question "How does one do homotopy theory in the category of smooth schemes over some field k?" Just as the category of smooth manifolds is too small to do classical homotopy theory, the category of smooth schemes over $\mathrm{k}$ is too small to do motivic homotopy theory. To fix this, we enlarge to the category $\Delta^{\mathrm{op}} \operatorname{Pre}\left((\mathrm{Sm} / \mathrm{k})_{\text {Nis }}\right)$ of simplicial Nisnievich presheaves. This allows us to do simplicial constructions as well as to impose a homotopy theoretic construction in which the affine line $\mathbb{A}^{1}$ plays the role of the unit interval. This theory was first constructed by Morel and Voedvosky in [MV99].

Our site is $(\mathrm{Sm} / \mathrm{k})_{\text {Nis. }}$. Here $\mathrm{Sm} / \mathrm{k}$ denotes smooth separated schemes over the field k. We give $\mathrm{Sm} / \mathrm{k}$ the Nisnievich topology; covers are étale covers such that over each point (possibly not closed) there is a point with the same residue field. The motivation behind using the Nisnevich topology, as opposed to say the Zariski topology, is that 
the Nisnevich cohomology is often the same as in the Zariski topology, but it can be computed using Čech cohomology.

For any object $X$ in a site $\mathcal{C}$, we have a representable presheaf $\mathcal{C}(\cdot, X)$. For each $Y \in \mathcal{C}$ this presheaf takes the value of the hom-set of morphisms in $\mathcal{C}$ from $Y$ to $X$.

Definition 1.1. We say that a site $\mathcal{C}$ is subcanonical if each representable presheaf of sets on $\mathcal{C}$ is a sheaf.

It turns out that the site of smooth schemes over $\mathrm{k}$ with the Nisnevich topology is subcanonical. Thus, we have an embedding $\mathrm{Sm} / \mathrm{k} \hookrightarrow \operatorname{Pre}(\mathrm{Sm} / \mathrm{k})$. To allow for simplicial constructions, we actually consider $\Delta^{\mathrm{op}} \operatorname{Pre}(\mathrm{Sm} / \mathrm{k})$.

Definition 1.2. The category of k-spaces is

$$
\operatorname{Spc}(\mathrm{k}):=\Delta^{\mathrm{op}} \operatorname{Pre}(\mathrm{Sm} / \mathrm{k})
$$

The site Sm/k has enough points, and so we are able to form stalks in $\operatorname{Spc}(\mathbf{k})$. Noting that the stalks are simplicial sets, we put a model structure on $\mathrm{Spc}(\mathrm{k})$ called the local model structure as follows:

Weak equivalences are maps of simplicial presheaves inducing equivalences of simplicial sets on all stalks

Cofibrations are the monomorphisms.

Fibrations Satisfy the right lifting property with respect to acyclic cofibrations.

It is a theorem of Jardine [Jar87] that this produces a proper closed simplicial model structure on $\operatorname{Spc}(k)$.

We define an object $Z$ in $\Delta^{\mathrm{op}}\left(\operatorname{Pre}(\mathrm{Sm} / \mathrm{k})_{\text {Nis }}\right)$ to be $\mathbb{A}^{1}$-local if for every projection map $X \times \mathbb{A}^{1} \stackrel{\pi}{\rightarrow} X$, the induced map $\operatorname{Hom}(X, Z) \stackrel{\operatorname{Hom}(\pi, Z)}{\longrightarrow} \operatorname{Hom}\left(X \times \mathbb{A}^{1}, Z\right)$ is an isomorphism. We then say that a morphism $P \stackrel{f}{\rightarrow} Q$ is a local $\mathbb{A}^{1}$ weak equivalence 
if the induced map $\operatorname{Hom}(Q, Z) \stackrel{\operatorname{Hom}(f, Z)}{\longrightarrow} \operatorname{Hom}(P, Z)$ is an isomorphism for each $\mathbb{A}^{1}$ local $Z$. Using Bousfield localization, we form a new model category, called the $\mathbb{A}^{1}$ homotopy category, which we abbreviate as $\mathcal{H}(\mathrm{k})$.

Let $X, Y \in \operatorname{Spc}(\mathrm{k})$ and $\operatorname{hom}(X, Y)$ be the simplicial set with $n$-simplices consisting of maps of simplicial presheaves $X \times \Delta^{n} \rightarrow Y$.

In the $\mathbb{A}^{1}$ homotopy category we can form pushouts and pullbacks, and so we are able to form wedge sums and smash products of pointed k-spaces.

\subsubsection{The bigraded family of spheres}

One of the important features of motivic homotopy theory is that it admits a bigraded family of spheres. There are two circles in the homotopy category, $S^{1}$ and $S^{\alpha}$. The circle $S^{1}$ can be formed as $\mathbb{A}^{1} / 0 \sim 1$ or as $\Delta^{1} / \partial \Delta^{1}$. As such, $S^{1}$ is best thought of as a topological circle. The circle $S^{\alpha}$ can be formed as $\mathbb{A}^{1} \backslash 0$, which is equivalent to $\mathbb{G}_{m}:=\operatorname{Spec}\left(\mathrm{k}\left[z, z^{-1}\right]\right)$. We refer to $S^{\alpha}$ as the geometric circle. We can form an $n+m \alpha$ dimensional sphere as the smash product of $n$ type $S^{1}$ circles and $m$ type $S^{\alpha}$ circles. It is well known that $S^{1} \wedge S^{\alpha} \simeq \mathbb{P}^{1}$.

In our notation, 1 and $\alpha$ correspond to the more standard notation $1=(1,0)$ and $\alpha=(1,1)$

\subsubsection{The stable motivic homotopy category}

Definition 1.3. A motivic prespectrum $X$ is a sequence of based k-spaces $X_{0}, X_{1}$, $X_{2}, \ldots$, along with structure maps $S^{1+\alpha} \wedge X_{N} \stackrel{\sigma}{\rightarrow} X_{N+1}$ satisfying the appropriate commutative diagrams. Each of the maps $\sigma$ is adjoint to a map $X_{N} \stackrel{\widetilde{\sigma}}{\rightarrow} \Omega^{1+\alpha} X_{N+1}$. If each of these maps is an equivalence, then we say that $X$ is a spectrum. Any prespectrum can be promoted to a spectrum in a canonical way.

Definition 1.4. Let $U \in \mathrm{Sm} / \mathrm{k}$ and $X$ be a motivic prespectrum. Then we define an 
inductive sequence

$$
\left[S^{m+n \alpha} \wedge U_{+}, X_{0}\right] \rightarrow\left[S^{m+n \alpha+(1+\alpha)} \wedge U_{+}, X_{1}\right] \rightarrow\left[S^{m+n \alpha+2(1+\alpha)} \wedge U_{+}, X_{2}\right] \rightarrow \ldots
$$

We define $\pi_{n+m \alpha} X(U)$ to be the colimit of the above sequence.

The following is a theorem of D. Dugger and D. Isaksen proved in [DI05].

Theorem 1.5. Consider the family of bigraded functors $\pi_{n+m \alpha}(-): \operatorname{Spt}(\mathrm{k}) \rightarrow \mathrm{Gp}$ defined by $\pi_{n+m \alpha} X:=\pi_{n+m \alpha} X(\operatorname{Spec}(\mathrm{k}))$. In the category of cellular $\mathrm{k}$ spectra these functors detect equivalences. For the definition of cellular, see definition 2.4.

Motivic spectra also produce (co)homology theories on smooth k-schemes is a way familiar to topologists.

Definition 1.6. Given a k-spectrum $\mathrm{E}$ and $U \in \mathrm{Sm} / \mathrm{k}$, we define the E-cohomology of $U$ by

$$
\mathrm{E}^{n+m \alpha}(U):=\left[U_{+}, \Sigma^{n+m \alpha} \mathrm{E}\right] .
$$

We define the $\mathrm{E}$ homology of $U$ by

$$
\mathrm{E}_{n+m \alpha}(U):=\left[S^{n+m \alpha}, \mathrm{E} \wedge U_{+}\right]
$$

By abuse of notation, we will write $\mathrm{E}^{n+m \alpha}$ and $\mathrm{E}_{n+m \alpha}$ whenever we mean $\mathrm{E}^{n+m \alpha}$ (Spec $\left.\mathrm{k}\right)$ and $\mathrm{E}_{n+m \alpha}($ Spec k) respectively.

\subsection{G-equivariant motivic homotopy theory}

Following [HKO11], let $(\mathrm{Sm} / \mathrm{k})_{\mathrm{Nis}_{G}}$ denote the site of $G$-equivariant smooth separated schemes over k with the Nisnievich topology, for $G$ a finite group. In our definition, the covers in the $G$-equivariant Nisnievich topology are $G$-equivariant étale maps $f$ in which for each point $x$ (in the scheme-theoretical sense) with isotropy 
group $H \subseteq G$, there exists a point in $f^{-1}(x)$ with the same residue field and the same isotropy group. By the category of based $G$-equivariant k-spaces we shall mean the category $\Delta^{\mathrm{op}} \operatorname{Pre}\left((\mathrm{Sm} / \mathrm{k})_{\mathrm{Nis}_{G}}\right)$ of pointed simplicial presheaves on the site $(\mathrm{Sm} / \mathrm{k})_{\mathrm{Nis}_{G}}$.

\subsubsection{The family of $C_{2}$ spheres}

In $C_{2}$-equivariant motivic homotopy theory we have four motivic circles. We have the two nonequivariant circles, $S^{1}$ and $S^{\alpha}$, by giving them the trivial action. We also have two $C_{2}$-equivariant circles $S^{\sigma}$ and $S^{\sigma \alpha}$. The circle $S^{\sigma}$ can defined as $\Delta^{1} / \partial \Delta^{1}$ with the action $z \mapsto-z$. The circle $S^{\sigma \alpha}$ will also be called $\mathbb{G}_{m}^{1 / z}$, defined as $\operatorname{Spec}\left(\mathrm{k}\left[z, z^{-1}\right]\right)$ equipped with an action $z \mapsto z^{-1}$. We can form $p+q \alpha+r \sigma+s \sigma \alpha$ spheres by smashing $p$ copies of $S^{1}$ with $q$ copies of $S^{\alpha}$ with $r$ copies of $S^{\sigma}$ with $s$ copies of $S^{\sigma \alpha}$. In particular, $S^{\sigma} \wedge S^{\sigma \alpha} \simeq \mathbb{P}^{1}$ with involution given by $z \mapsto-z^{-1}$. We denote this space by $\mathbb{P}_{-}^{1}$. We form the $C_{2}$-equivariant stable category by stabilizing with respect to $\mathbb{P}^{1} \wedge \mathbb{P}_{-}^{1}$. We will sometimes denote $\mathbb{P}^{1} \wedge \mathbb{P}_{-}^{1}$ by $\mathbb{T}_{G}$.

As an aside, I would like to point out that the authors of [HKO11] use the greek letter $\gamma$ instead of $\sigma$. The reason for this difference is an aesthetic one, although $\sigma$ is also used in [HVØ16] in place of $\gamma$. However, in [HVØ16] the authors use a Voevodsky type grading. I prefer the grading convention of [HKO11].

\subsubsection{Two kinds of classifying spaces}

Recall that topologically, the classifying space $B G$ for a group $G$ is constructed by taking the quotient of a $G$-free contractable $G$-CW-complex $E G$ by the group $G$. Topologically, all such constructions are equivalent. However, this is not the case motivically. Motivically, there are two different constructions of the classifying space $B G$; there is the usual simplical construction, and there is a geometric classifying space construction which can be found in [MV99, Tot99]. I will denote the usual simplicial model of the free contractible $G$ space by $E G$, and I will denote the geometric 
construction by $E_{e t} G$. We will concern ourselves explicitly with the group $G=C_{2}$, and so I give an explicit model for $E_{e t} C_{2}$.

Definition 1.7. Consider the spaces $\mathbb{A}^{n} \backslash 0$ pointed at 1 with a $C_{2}$ action given by $z \mapsto-z$. We have natural inclusions

$$
\mathbb{A}^{n} \backslash 0 \subseteq \mathbb{A}^{n+1} \backslash 0
$$

for each $n$. Therefore, we can form a space $\mathbb{A}^{\infty} \backslash 0$, which we call $E_{e t} C_{2}$, in the obvious way. We can also form a space $B_{e t} C_{2}$ by forming the quotient $\mathbb{A}^{n} \backslash 0 / C_{2}$ for each $n$ and then taking the direct limit of the spaces with respect to inclusion.

In [Voe03], V. Voevodsky computes the motivic $\mathbb{Z} / 2$ cohomology of $B_{e t} C_{2}$.

Proposition 1.8. The algebra structure of the motivic $\mathbb{Z} / 2$ cohomology of $B_{e t} C_{2}$ is

$$
H^{\star}\left(B_{e t} C_{2} ; \mathbb{Z} / 2\right) \cong H^{\star}[a, b] /\left(a^{2}-\tau b\right)
$$

Here $\tau$ is the tate twist of degree $\alpha-1$, a the cohomology class of degree $\alpha$, and $b$ the cohomology class of degree $1+\alpha$.

\subsection{G-equivariant stable motivic homotopy theory}

Classically, the tools of stable $G$-equivariant stable homotopy theory are contained in a paper by Greenlees and May [GM95]. Those tools involve a cofibration sequence called the tate diagram, a certain adams isomorphism saying that fixed points and qoutients behave well after smashing with a free contractible space $E G$, and a certain geometric fixed point functor $\Phi^{G}$. The geometric fixed point functor $\Phi^{G}$ is supposed to be the naive notion of taking the fixed points of a spectrum $E$ which is implemented by taking fixed points at the prespectrum level. We can do the same construction motivically. 
Definition 1.9. Let $\mathrm{E}$ be a $G$ equivariant motivic spectrum defined at the prespectrum level by a sequence of spaces $X_{0}, X_{1}, X_{2}, \ldots$. Then we can define a nonequivariant motivic spectrum $\Phi_{e t}^{G}(\mathrm{E})$. The prespectrum is formed by the sequence of spaces $Y_{n}:=\left(X_{n}\right)^{G}$. The structure maps are then defined by taking $G$ fixed points on the structure maps $\mathbb{T}_{G} \wedge X_{n} \stackrel{\sigma}{\rightarrow} X_{n+1}$. Since $\left(\mathbb{T}_{G}\right)^{G}=\mathbb{P}^{1}$, we obtain structure maps $\mathbb{P}^{1} \wedge Y_{n} \stackrel{\sigma_{\text {fixed }}}{\longrightarrow} Y_{n+1}$.

Another important tool of equivariant homotopy theory is the Tate diagram. Classically this is given by a cofibration sequence $E G_{+} \rightarrow S^{0} \rightarrow \widetilde{E G}$ where $E G:=$ $|B(G, G, *)|$. Smashing this with a $G$ equivariant spectrum $\mathrm{E}$ we can form an equivariant cofibration

$$
E G_{+} \wedge \mathrm{E} \rightarrow \mathrm{E} \rightarrow \widetilde{E G} \wedge \mathrm{E}
$$

Taking $G$ fixed points we obtain a diagram

$$
E G_{+} \wedge_{G} \mathrm{E} \rightarrow \mathrm{E}^{G} \rightarrow \Phi^{G}(\mathrm{E}) .
$$

Classically, an equivalent approach involves considering the reduced regular suspension $V$ of the group $G$. Set $S(n V):=V^{\oplus n} \backslash 0$. We may then form cofibration sequences

$$
S(n V)_{+} \rightarrow S^{0} \rightarrow S^{n V}
$$

Taking the colimit, we obtain an equivariant cofibration

$$
S(\infty V)_{+} \rightarrow S^{0} \rightarrow S^{\infty V}
$$

Smashing the Tate diagram with a $G$-equivariant spectrum E, we can form a $G$ - 
equivariant cofibration sequence

$$
S(\infty V)_{+} \wedge \mathrm{E} \rightarrow \mathrm{E} \rightarrow S^{\infty V} \wedge \mathrm{E}
$$

Topologically, eq. (1.1) and eq. (1.2) are equivalent constructions. Unfortunately this is not the case motivically. In the motivic universe, $\left(S^{\infty V} \wedge \mathrm{E}\right)^{G}=\Phi^{G}(\mathrm{E})$. Unfortunately, it is not true that $(\widetilde{E G} \wedge \mathrm{E})^{G}=\left(S^{\infty V} \wedge \mathrm{E}\right)^{G}$ in general. This is easy to see in the case $G=C_{2}$. Since in this case $S^{\infty V}=S^{\infty \sigma+\infty \sigma \alpha}$, smashing it with E kills both of the equivariant suspensions $\sigma$ and $\sigma \alpha$, which we would expect to happen. However, $\left|B\left(C_{2}, C_{2}, *\right)\right|$ is a model for $S^{\infty \sigma}$, and so we only kill the $\sigma$ suspensions but not the $\sigma \alpha$ suspensions whenever we smash with $\mathrm{E}$.

Motivically, the following two cofiber sequences of pointed $C_{2}$ k-spaces are useful for computational purposes,

$$
\begin{gathered}
C_{2+} \rightarrow S^{0} \rightarrow S^{\sigma}, \\
(\mathbb{A}(n \sigma) \backslash 0)_{+} \rightarrow S^{0} \rightarrow S^{n \sigma+n \sigma \alpha} .
\end{gathered}
$$

Here $\mathbb{A}(n \sigma)$ denotes the affine $n$-space $\mathbb{A}^{n}$ with $C_{2}$ action $z \mapsto-z$. These two cofiber sequences induces the cofiber sequences

$$
E C_{2+} \rightarrow S^{0} \rightarrow \widetilde{E C_{2}}
$$

and

$$
E_{e t} C_{2+} \rightarrow S^{0} \rightarrow \widetilde{E_{e t} C_{2}}
$$

respectively. $\widetilde{E_{e t} C_{2}}$ is a model for $S^{\infty \sigma+\infty \sigma \alpha}$, and so it follows that

$$
\left(X \wedge \widetilde{E_{e t} C_{2}}\right)^{C_{2}} \cong \Phi_{e t}^{C_{2}}(X)
$$


Equivariant stable motivic homotopy theory was first introduced in [HKO11] as a tool for solving Thomason's homotopy limit problem for algebraic Hermitian K-theory. The authors of that paper constructed the $C_{2}$-equivariant motivic spectrum MGLR, called algebraic Hermitian cobordism. In the process, they also proposed the spectrum $\Phi^{C_{2}}$ (MGLR) as a motivic analogue of the (topological) unoriented cobordism spectrum MO. In the present thesis, I modify this definition by putting MGLO $=\Phi_{e t}^{C_{2}}(\mathrm{MGLR})$. One of my main results is calculating the coefficients of MGLO. In particular, I prove: Theorem 1.10. MGLO is a wedge of suspensions of $H \mathbb{Z} / 2_{\text {Mot }}$. In particular, the coefficients of MGLO are direct sums of Bloch Chow groups of the ground field with coefficients $\mathbb{Z} / 2$.

A more precise statement is given in theorem 2.17 below. In chapter III, I also extend these results to a motivic analogue of oriented cobordism, and in chapter IV, I calculate the coefficients of a certain localization of MGLR. 


\section{CHAPTER II}

\section{A motivic analogue of $\mathrm{MO}$}

In section 1 of this chapter, we give a detailed account of how to construct the motivic spectrum MGLO. In section 2, we give a full computation of the coefficients of this spectrum up to knowledge of the coefficients of motivic $H \mathbb{Z} / 2$. In particular, our computation gives an explicit description of the $\mathbb{Z} / 2$-algebra structure of the coefficients of MGLO over the fields $\mathbb{R}$ and $\mathbb{C}$. Moreover, the topological realization of MGLO over the field $\mathbb{C}$ is $\mathrm{MO}$.

\subsection{The construction of MGLO}

The idea behind our definition of MGLO is that, just as the geometric fixed points of $\mathrm{MO}$ is $\mathrm{MR}$, the geometric fixed points of MGLR should be MGLO. The definition presented here is different than the definition given in [HKO11]. Using simplicial $E C_{2}$, the authors of [HKO11] define,

$$
\mathrm{MGLO}:=\left(\widetilde{E C_{2}} \wedge \mathrm{MGLR}\right)^{C_{2}}
$$

However, this definition does not satisfy a crucial property. Topologically, given a $G$-equivariant spectrum $\mathrm{E}$, the functor $\Phi^{G}(-):=(-\wedge \widetilde{E G})^{G}$ applied to $\mathrm{E}$ produces a nonequivariant spectrum $\Phi^{G}(\mathrm{E})$ which is equivalent to forgetting $\mathrm{E}$ to the prespec- 
trum level and then simultaneously taking $G$-fixed points of the spaces making up the prespectrum of $E$ and the connecting maps to form a nonequivariant prespectrum. One can then promote this to a nonequivariant spectrum in the usual way. Similarly, in our definition, MGLO is defined by forgetting MGLR to the level of prespectra and then taking $C_{2}$-fixed points of the spaces and connecting maps to form a nonequivariant prespectrum. Promoting this to a spectrum defines MGLO.

This alternative definition of MGLO turns out to be different than eq. (2.1). The reason being that simplicial $\widetilde{E C_{2}}$ is a model for $S^{\infty \sigma}$. This only takes into account the $\sigma$-grading. However, we need to also take into account the $\sigma \alpha$ grading. In other words, our $\widetilde{E C_{2}}$ should really be a model of $S^{\infty \sigma+\infty \sigma \alpha}$. It turns out that the 1-point compactification of geometric $E C_{2}$ serves as a model, and we have that,

$$
\mathrm{MGLO} \simeq\left(\mathrm{MGLR} \wedge S^{\infty \sigma+\infty \sigma \alpha}\right)^{C_{2}}
$$

\subsubsection{Quadratic forms}

Following [HKO11, Section 6.1], we consider the hyperbolic quadratic form on $\mathrm{k}^{2 n}$ :

$$
q\left(x_{1}, \ldots, x_{2 n}\right)=x_{1} x_{2}+\ldots+x_{2 n-1} x_{2 n} .
$$

The associated symmetric bilinear form is

$$
b\left(\left(x_{1}, \ldots, x_{2 n}\right),\left(y_{1}, \ldots, y_{2 n}\right)\right)=\sum_{i=1}^{n} x_{2 i} y_{2 i-1}+x_{2 i-1} y_{2 i} .
$$

The $b$-adjoint of a matrix $A=\left(a_{i, j}\right)_{i, j=0}^{2 n}$ is a $2 n \times 2 n$ matrix $A^{T_{b}}$ such that

$$
b(A x, y)=b\left(x, A^{T_{b}} y\right) .
$$


Explicitly, putting $A^{T_{b}}=\left(b_{i, j=1}^{2 n}\right)$, one has

$$
\begin{aligned}
& b_{2 i, 2 j}=a_{2 j-1,2 i-1} \\
& b_{2 i-1,2 j-1}=a_{2 j, 2 i} \\
& b_{2 i, 2 j-1}=a_{2 j, 2 i-1} \\
& b_{2 i-1,2 j}=a_{2 j-1,2 i}
\end{aligned}
$$

Notice that there is a $C_{2}$ action on the quadric

$$
\mathcal{Q}_{n}:=\mathbb{V}(x, y \mid b(x, y)=1)
$$

where $\mathbb{V}\left(x_{i} \mid E\right)$ (sometimes abbreviated to $\mathbb{V}(E)$ ) denotes the locus of the equations $E$ in the variables $x_{i}$, given by

$$
x \leftrightarrow y
$$

Taking $C_{2}$ fixed points of the quadric under this action, we have:

$$
\left(\mathcal{Q}_{n}\right)_{C_{2}}=\mathbb{V}(x, y \mid b(x, y)=1, x=y)=\mathbb{V}\left(\sum_{i=1}^{n} x_{2 i} y_{2 i-1}+x_{2 i-1} y_{2 i}-1, x=y\right)
$$

The projection from eq. (2.3) onto the $x$ coordinate scaled by a factor of 2 gives an equivalence to $Q_{2 n-1}:=\mathbb{V}\left(x \in \mathrm{k}^{2 n} \mid x_{1} x_{2}+x_{3} x_{4}+\ldots+x_{2 n-1} x_{2 n}-1\right)$. But the projection from eq. (2.3) onto the $x$-axis gives the same thing as projecting $\mathcal{Q}_{n}$ onto the $x$-axis. So long as $x \neq 0$ there exists a $y$ such that $b(x, y)=1$. But this means that the image of the projection map is $\mathbb{A}^{2 n} \backslash 0$. It is a standard result that $\mathbb{A}^{2 n} \backslash 0$ has the homotopy type of $S^{2 n-1, n}=S^{n-1+n \alpha}$. Now returning to eq. (2.2) we will define 
the even dimensional orthogonal groups by

$$
O_{2 n}:=\left\{A \in G L_{2 n}(k) \mid A A^{T_{b}}=I\right\} .
$$

The group $O_{2 n}$ acts on the quadric $Q_{2 n-1}$ in a natural way. We can write $Q_{2 n-1}$ as

$$
\mathbb{V}\left(\frac{b(x, x)}{2}-1\right) .
$$

The action on $Q_{2 n-1}$ is given element-wise by $A \cdot x=A x$. Notice that

$$
b(A x, A x)=b\left(x, A^{T_{b}} A x\right)=b(x, x) .
$$

Therefore we have defined an $O_{2 n}$ action on $Q_{2 n-1}$. We define $O_{2 n-1}$ to be

$$
O_{2 n-1}:=\left\{A \in O_{2 n} \mid A\langle 1,1,0, \ldots, 0\rangle=\langle 1,1,0, \ldots, 0\rangle\right\} .
$$

For brevity we will write $x^{0}$ in place of $\langle 1,1,0, \ldots, 0\rangle$. It is not out of place to ask if our definition for $O_{2 n-1}$ is a good one. If we would have defined $O_{2 n-1}$ to be matrices $A \in O_{2 n}$ such that $A e_{1}=e_{1}$ then it is clear that $a_{1 j}=a_{j 1}=\delta_{i j}$ if one writes down what is going on. This gives the only restrictions on $O_{2 n-1}$ other than those induced from the ambient group $O_{2 n}$, and so $O_{2 n-1}$ would have a natural inclusion into $O_{2 n}$. For the vector $\langle 1,1,0, \ldots, 0\rangle$ things are not so clear, but we do know that there is a transition matrix (though not necessarily unique) from the point $\langle 1,1,0, \ldots, 0\rangle$ to $e_{1}$. Therefore, the subgroup of matrices fixing the point $\langle 1,1,0, \ldots, 0\rangle$ is isomorphic to the subgroup of matrices fixing $e_{1}$. So it makes sense to identify $O_{2 n-1}$ as we have above.

Lemma 2.1. $O_{2 n}$ acts transitively on $Q_{2 n-1}$ and the fixed point subgroup of $\langle 1,1,0, \ldots, 0\rangle$ is $O_{2 n-1}$.

Proof. For the transitivity claim we need to show that for $x, y \in Q_{2 n-1}$ there is 
some $A \in O_{2 n}$ such that $A x=y$. Note that it is enough to show that for any $x \in Q_{2 n-1}$ there exists a matrix $A \in O_{2 n}$ such that $A x=x^{0}$. For if $A x=x^{0}$ and $B y=x^{0}$, we have that $B^{-1} A x=y$. Consider the orthonormal basis $\mathcal{B}_{1}$ given by $\left\{\frac{\sqrt{2}}{2} x^{0}, \frac{\sqrt{2}}{2}\langle 1,-1,0, \ldots, 0\rangle, e_{3}, \ldots, e_{2 n}\right\}$, and an orthonormal basis $\mathcal{B}_{2}$ given by $\left\{\frac{x}{\|x\|}, v_{2}, \ldots, v_{2 n}\right\}$. Then there exists a change of basis matrix $P$ from $\mathcal{B}_{2}$ to $\mathcal{B}_{1}$ which, in particular, sends $\frac{x}{\|x\|}$ to $\frac{\sqrt{2}}{2} x^{0}$. This then implies that $P x=\lambda x^{0}$ for some $\lambda \in \mathrm{k}$. But if $x \in Q_{2 n-1}$, then $\frac{b(x, x)}{2}=\frac{b(P x, P x)}{2}=\frac{b\left(\lambda x^{0}, \lambda x^{0}\right)}{2}=\lambda^{2} \frac{b\left(x^{0}, x^{0}\right)}{2}=\lambda^{2}=1$. Therefore $\lambda= \pm 1$. Suppose that $\lambda=-1$. Then $P x=-x^{0} \Rightarrow(-P) x=x^{0}$. But $-P \in O_{2 n}$. This proves the transitivity claim. The claim about $O_{2 n-1}$ is true by definition.

We define,

$$
\begin{array}{r}
Q_{2 n-2}:=\mathbb{V}\left(x \in \mathrm{k}^{2 n} \mid b\left(x, x^{0}\right), b(x, x)+1\right) \\
=\left\{x \in \mathrm{k}^{2 n} \mid x_{1} x_{2}+\ldots+x_{2 n-1} x_{2 n}+1=x_{1}+x_{2}=0\right\} .
\end{array}
$$

We would like to make analogous statements to lemma 2.1 for $O_{2 n-1}$ and $Q_{2 n-2}$. First, however, I will show that $Q_{2 n-2}$ is homotopy equivalent to a familiar space.

Lemma 2.2. $Q_{2 n-2}$ is homotopy equivalent to $S^{2 n-1, n-1}=S^{n-1+(n-1) \alpha}$.

Proof. We have that

$$
Q_{2 n-2}=\mathbb{V}\left(x \in \mathrm{k}^{2 n} \mid x_{1} x_{2}+\ldots+x_{2 n-1} x_{2 n}+1, x_{1}+x_{2}\right) .
$$

We note that this space is homotopy equivalent to

$$
\mathbb{V}\left(\left(y, x_{3}, x_{4}, \ldots, x_{2 n}\right) \in \mathrm{k}^{2 n-1} \mid-y^{2}+x_{3} x_{4}+\ldots+x_{2 n-1} x_{2 n}+1\right) .
$$

But this is easily seen to equivalent to

$$
\operatorname{Spec}\left(\mathbf{k}\left[y, x_{3}, x_{4}, \ldots, x_{2 n-1}, x_{2 n}\right] /\left((1-y)(1+y)+x_{3} x_{4}+\ldots+x_{2 n-1} x_{2 n}\right)\right)
$$


Now, by [ADF14, Theorem 2], we notice that

$$
S^{n-1+(n-1) \alpha} \simeq \operatorname{Spec}\left(\mathrm{k}\left[z, a_{3}, a_{4}, \ldots, a_{2 n-1}, a_{2 n}\right] /\left(a_{3} a_{4}+\ldots+a_{2 n-1} a_{2 n}-z(1+z)\right) .\right.
$$

Using the change of variables $z \mapsto-\frac{1}{2}(1+y), a_{i} \mapsto \frac{1}{2} x_{i}$, we have that

$$
\begin{array}{r}
\operatorname{Spec}\left(\mathrm{k}\left[z, a_{3}, a_{4}, \ldots, a_{2 n-1}, a_{2 n}\right] /\left(a_{3} a_{4}+\ldots+a_{2 n-1} a_{2 n}-z(1+z)\right)\right. \\
\simeq \operatorname{Spec}\left(\mathrm{k}\left[y, x_{3}, x_{4}, \ldots, x_{2 n-1}, x_{2 n}\right] /\left(\frac{1}{4}\left(x_{3} x_{4}+\ldots+x_{2 n-1} x_{2 n}+(1-y)(1+y)\right)\right)\right. \\
\simeq \operatorname{Spec}\left(\mathrm{k}\left[y, x_{3}, x_{4}, \ldots, x_{2 n-1}, x_{2 n}\right] /\left(x_{3} x_{4}+\ldots+x_{2 n-1} x_{2 n}+(1-y)(1+y)\right)\right) .
\end{array}
$$

The $O_{2 n}$ action on $Q_{2 n-1}$ induces an $O_{2 n-1}$ action on $Q_{2 n-2}$. Recall that $O_{2 n-1}$ acts point-wise on the quadric $Q_{2 n-2}$ by $A \cdot x \mapsto A x$. Notice that $Q_{2 n-2}$ is induced from the form $b_{2 n}(x, y) . x \in Q_{2 n-2}$ implies that $\frac{b_{2 n}(x, x)}{2}=-1$. Since

$$
b(A x, A x)=b\left(x, A^{T_{b}} A x\right)=b(x, x),
$$

it only remains to show that if $x_{1}=-x_{2}$ and $y=\left(y_{1}, y_{2}, \ldots, y_{2 n}\right)$ is the image of $x$, then $y_{1}=-y_{2}$. But notice that for $x \in Q_{2 n-2}$ we have that $b(x,\langle 1,1,0, \ldots, 0\rangle)=0$. Let $A \in O_{2 n-1}$ and let $y=\left\langle y_{1}, y_{2}, \ldots, y_{2 n}\right\rangle$ be the image of $x$. Then,

$$
\begin{array}{r}
y_{1}+y_{2}=b(y,\langle 1,1,0, \ldots, 0\rangle)=b(A x,\langle 1,1,0, \ldots, 0\rangle)=b\left(x, A^{T_{b}}\langle 1,1,0, \ldots, 0\rangle\right) \\
=b(x,\langle 1,1,0, \ldots, 0\rangle)=x_{1}+x_{2}=0 .
\end{array}
$$

This proves that $O_{2 n-1}$ acts on the quadric $Q_{2 n-2}$.

Lemma 2.3. $O_{2 n-1}$ acts transitively on $Q_{2 n-2}$ and the fixed point subgroup of $y^{0}=\langle 1,-1,0, \ldots, 0\rangle$ can be naturally identified with $O_{2 n-2}$.

Proof. We prove the transitivity claim in a similar manner to lemma 2.1. It will 
be enough to show that for any $x \in Q_{2 n-2}$ there is a matrix $A \in O_{2 n-1}$ such that $A x=y^{0}$.

Notice that technically our $O_{2 n-1}$ lives inside of $O_{2 n}$. We choose an orthonormal basis $\mathcal{B}_{1}=\left\{\frac{x^{0}}{\left\|x^{0}\right\|}, \frac{y^{0}}{\left\|y^{0}\right\|}, e_{3}, \ldots, e_{2 n}\right\}$, and $\mathcal{B}_{2}=\left\{\frac{x^{0}}{\left\|x^{0}\right\|}, \frac{x}{\|x\|}, v_{3}, \ldots, v_{2 n}\right\}$. Then there exists

a change of basis matrix $P$ from $\mathcal{B}_{2}$ to $\mathcal{B}_{1}$ which sends $x^{0}$ to $x^{0}$ and $x$ to $\frac{y^{0}}{\left\|y^{0}\right\|}$. This implies that for $x \in Q_{2 n-2}$ we have that $P x=\lambda y_{0}$. We have that

$$
-1=\frac{b(x, x)}{2}=\frac{b(P x, P x)}{2}=\frac{b\left(\lambda y^{0}, \lambda y^{0}\right)}{2}=\frac{\lambda^{2} b\left(y^{0}, y^{0}\right)}{2}=-\lambda^{2} \Rightarrow \lambda= \pm 1 .
$$

If $\lambda=1$ then we are done. If $\lambda=-1$ then we have that $(-P) x=y^{0}$. This proves the transitivity claim.

The subgroup of $O_{2 n-1}$ which fixes the point $y^{0}=\langle 1,-1,0, \ldots, 0\rangle \in \mathbf{k}^{2 n}$ is,

$$
\left\{A \in O_{2 n} \mid A x^{0}=x^{0} \text { and } A y^{0}=y^{0}\right\}=\left\{A \in O_{2 n-1} \mid A e_{1}=e_{1} \text { and } A e_{2}=e_{2}\right\} .
$$

But this is just matrices $A \in O_{2 n}$ of the form:

$$
A=\left[\begin{array}{ccccc}
1 & 0 & 0 & \ldots & 0 \\
0 & 1 & 0 & \ldots & 0 \\
0 & 0 & x_{3,3} & \ldots & x_{3,2 n} \\
\vdots & \vdots & \vdots & \ddots & \vdots \\
0 & 0 & x_{2 n, 3} & \ldots & x_{2 n, 2 n}
\end{array}\right]
$$

This shows that $O_{2 n-2}$ can be naturally identified with the subgroup of $O_{2 n-1}$ which fixes the point $y^{0}$.

\subsubsection{Cellularity}

The following definition is due to [DI05, Definition 2.1].

Let $\mathcal{M}$ be a pointed model category, and let $\mathcal{A}$ be a set of objects in $\mathcal{M}$. 
Definition 2.4. The class of $\mathcal{A}$-cellular objects is the smallest class of objects of $\mathcal{M}$ such that

1. every object of $\mathcal{A}$ is $\mathcal{A}$-cellular;

2. if $X$ is weakly equivalent to an $\mathcal{A}$-cellular object, then $X$ is cellular;

3. if $\mathcal{D}: I \rightarrow \mathcal{M}$ is a diagram such that $\mathcal{D}$ is $\mathcal{A}$-cellular, then so is hocolim $\mathcal{D}$.

Choosing $\mathcal{M}$ to be the stable motivic homotopy category, and choosing $\mathcal{A}$ to be the motivic sphere spectrum, we obtain the cellular stable motivic homotopy category. Adapting the proof of [DI05, Proposition 4.1], I will prove the following.

Proposition 2.5. The variety $O_{n}$ is stably cellular for every $n \geq 1$.

Proof. We first suppose that $n=2 k$. Let $x=\langle 1,1,0, \ldots, 0\rangle$. Now consider the fiber bundle $O_{n} \rightarrow \mathbb{P}^{n-1}$ given by

$$
O_{n} \stackrel{m_{x}}{\rightarrow} \mathbb{A}^{n} \rightarrow \mathbb{A}^{n} / \mathbb{A}^{n} \backslash 0 \simeq \mathbb{P}^{n-1}
$$

Here $m_{x}$ denotes the map $A \mapsto A x$. Notice that $m_{x}$ induces a transitive action of $O_{n}$ on the motivic sphere $Q_{n-1}$. The fiber over the point $[1,0,0, \ldots, 0]$ consists of all $A \in O_{n}$ such that $a_{11} \neq 1$, and $a_{j 1}=0$ for $j \geq 2$. Recall that

$$
O_{n-1} \cong\left\{A \in O_{n} \mid A\langle 1,0,0, \ldots\rangle=\langle 1,0,0, \ldots\rangle\right\}
$$

But this is just $\left\{A \in m_{x}^{-1}([1,0,0, \ldots]) \mid a_{11}=1\right\}$. Since $\operatorname{det}\left(A A^{T}\right)=\operatorname{det}(A) \operatorname{det}\left(A^{T}\right)=$ $\operatorname{det}(A)^{2}=1$, it follows that $a_{11}= \pm 1$, and so $m_{x}^{-1}([1,0,0, \ldots])=O_{n-1} \times\{ \pm 1\}$. As a scheme, but not as a group, this is isomorphic to

$$
\{ \pm 1\} \times \mathbb{A}^{n-1} \times O_{n-1}
$$


which is stably cellular by induction and [DI05, Lemma 3.4]. The usual cover of $\mathbb{P}^{n}$ by affines is a completely trivializing cover for the bundle, so [DI05, Lemma 3.8] applies.

\subsubsection{Two-sided bar construction}

Recall that we have the following equivalences,

$$
Q_{n} \simeq\left\{\begin{array}{l}
S^{k+k \alpha} \text { if } n=2 k \\
S^{k-1+k \alpha} \text { if } n=2 k-1
\end{array}\right.
$$

The groups $O_{n}$ act on the quadrics $Q_{n-1}$, allowing us to form the two-sided bar construction, which we now discuss.

Let $G$ be a finite group and $X$ and $Y$ motivic spaces. If $X \times G \rightarrow X$ is a right $G$ action and $G \times Y \rightarrow Y$ is a left $G$ action, then we form the two sided bar construction $B(X, G, Y)$ as the left derived functor of the coequalizer of $X \times G \times Y \rightrightarrows X \times Y$. We denote the geometric realization of $B(X, G, Y)$ by $|B(X, G, Y)|$.

Definition 2.6. In the special case $X=Y=*$, we define $B G:=|B(*, G, *)|$.

Lemma 2.7. $\left|B\left(O_{n}, O_{n-1}, *\right)\right| \simeq Q_{n-1}$.

Proof. It is well known for $H \hookrightarrow G$ an inclusion of groups that the left coset $G / H$ is isomorphic to $|B(G, H, *)|$. Taking $G=O_{n}$ and $H=O_{n-1}$, this gives

$$
O_{n} / O_{n-1} \cong\left|B\left(O_{n}, O_{n-1}, *\right)\right|
$$

Notice that by the above discussion, $O_{n}$ acts on $Q_{n-1}$, and the stabilizer of a point is $O_{n-1}$. This induces an isomorphism between $O_{n} / O_{n-1}$ and $Q_{n-1}$, proving that

$$
Q_{n-1} \cong\left|B\left(O_{n}, O_{n-1}, *\right)\right|
$$


Lemma 2.8. $|B(G, G, *)| \simeq *$. In particular, we have $\left|B\left(O_{n}, O_{n}, *\right)\right| \simeq *$.

Proof. $* \cong G / G \cong|B(G, G, *)|$.

Proposition 2.9. $\left|B\left(*, O_{n}, Q_{n-1}\right)\right| \simeq B O_{n-1}$.

Proof. We have that $\left|B\left(*, O_{n}, Q_{n-1}\right)\right| \simeq\left|B\left(*, O_{n},\left|B\left(O_{n}, O_{n-1}, *\right)\right|\right)\right| \simeq$ $\left|B\left(\left|B\left(*, O_{n}, O_{n}\right)\right|, O_{n-1}, *\right)\right| \simeq\left|B\left(*, O_{n-1}, *\right)\right|$.

\subsubsection{The prespectrum for MGLO}

The identifications from Theorem proposition 2.9 imply that we have a map

$$
B O_{n-1} \stackrel{\pi}{\rightarrow} B O_{n}
$$

which is built from gluing together face maps which are projections,

$$
\underbrace{O_{n} \times O_{n} \times \ldots \times O_{n}}_{m \text { times }} \times Q_{n-1} \rightarrow \underbrace{O_{n} \times O_{n} \times \ldots \times O_{n}}_{m \text { times }} .
$$

Therefore, we can think of eq. (2.7) as a sphere bundle. This allows us to define Thom space like objects as the homotopy cofiber of $\pi$. That is, the Thom space of $B O_{n}$, denoted Thom $\left(B O_{n}\right)$, is defined by the homotopy pushout;

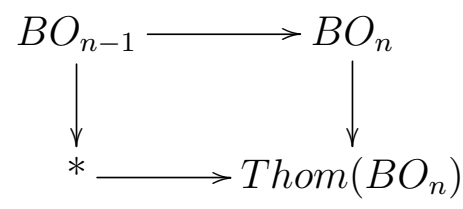

The spaces Thom $\left(\mathrm{BO}_{2 n}\right)$ will form the spaces for the prespectrum of MGLO. Now we discuss how to define the connecting maps. 
Definition 2.10. Notice that the natural inclusions $O_{n-1} \times O_{m-1} \subset O_{n} \times O_{m}$ induce maps $B\left(O_{n-1} \times O_{m-1}\right) \rightarrow B\left(O_{n} \times O_{m}\right)$. We define

$$
\operatorname{Thom}\left(B\left(O_{n} \times O_{m}\right)\right):=B\left(O_{n} \times O_{m}\right) / B\left(O_{n-1} \times O_{m-1}\right) \text {. }
$$

It is clear that $\operatorname{Thom}\left(B\left(O_{2 r} \times O_{2 s}\right)\right) \simeq \operatorname{Thom}\left(B O_{2 r}\right) \wedge \operatorname{Thom}\left(B O_{2 s}\right)$.

The even dimensional thom spaces Thom $\left(\mathrm{BO}_{2 n}\right)$ form the terms of the prespectrum. Since $\mathbb{G}_{m} \simeq S_{2} \subset O_{2}$ by proposition 3.1, we get the canonical map

$$
\mathbb{P}^{1} \simeq \Sigma \mathbb{G}_{m} \rightarrow B O_{2} .
$$

We can then define the structure maps by

$$
\begin{array}{r}
\mathbb{P}^{1} \wedge \operatorname{Thom}\left(B_{2 n}\right) \rightarrow \operatorname{Thom}\left(B_{2}\right) \wedge \operatorname{Thom}\left(B_{2 n}\right) \stackrel{\simeq}{\rightarrow} \operatorname{Thom}\left(B\left(O_{2} \times O_{2 n}\right)\right) \\
\rightarrow \operatorname{Thom}\left(B O_{2 n+2}\right) .
\end{array}
$$

Thus we have defined a prespectrum and so we can promote it to a spectrum in the usual way. This defines the spectrum MGLO.

Notice that since the orthogonal groups are stably cellular by proposition 2.5, it follows that the classifying spaces $B O_{n}$ is also stably cellular. Since each of the thom spaces Thom $\left(B O_{n}\right)$ are constructed as the homotopy cofiber of the inclusion $B O_{n-1} \rightarrow B O_{n}$, it follows that the spaces $\operatorname{Thom}\left(B O_{n}\right)$ are also cellular. Since these are the spaces defining the prespectrum of MGLO, it follows that MGLO is cellular. 


\subsection{Computing the coefficients of MGLO}

Combining proposition 2.9 with a Mayer-Vietoris argument as in [MS16] gives us the following Thom isomorphisms in motivic $H \mathbb{Z} / 2$ (co)homology.

$$
\begin{aligned}
H^{\star}\left(B O_{n+}\right) \cong H^{\star+\omega_{n}}\left(\operatorname{Thom}\left(B O_{n}\right)\right) \\
H_{\star}\left(B O_{n+}\right) \cong H_{\star+\omega_{n}}\left(\operatorname{Thom}\left(B O_{n}\right)\right)
\end{aligned}
$$

Here $\omega_{2 k}:=k+k \alpha$ and $\omega_{2 k+1}:=k+1+k \alpha$.

For each space $B O_{n}$, we get a unique Thom class $T h o m\left(B O_{n}\right) \stackrel{w_{n}}{\longrightarrow} \Sigma^{\omega_{n}} H \mathbb{Z} / 2$. Composing $w_{n}$ with the homotopy cofiber of the map $B O_{n-1_{+}} \rightarrow B O_{n+}$, we get a class $w_{n} \in H^{\omega_{n}}\left(B O_{n+}\right)$. The following theorem has essentially been proved by A. Smirnov and A. Vishik in [SV14] using different language from the present paper. The biggest difference between [SV14] and the theorem presented here is that [SV14] only applies to fields of characteristic 0 for which $\sqrt{-1} \in \mathrm{k}$, whereas the present theorem holds for any field $\mathrm{k}$ of characteristic 0 .

Theorem 2.11. There are a unique set of classes $w_{1}, w_{2}, \ldots, w_{n}$ belonging to motivic $\mathbb{Z} / 2$ cohomology for which,

$$
H^{\star}\left(B O_{n+}\right) \cong H^{\star}\left[w_{1}, \ldots, w_{n}\right]
$$

Here $\operatorname{deg}\left(w_{2 i}\right)=i+i \alpha$ and $\operatorname{deg}\left(w_{2 i+1}\right)=i+1+i \alpha$.

Proof. Notice that the cofibration $\mathrm{BO}_{n-1_{+}} \rightarrow \mathrm{BO}_{n_{+}} \rightarrow$ Thom $\left(B O_{n}\right)$ induces a long exact sequence in cohomology given by

$$
\ldots \rightarrow H^{\star}\left(\operatorname{Thom}\left(B O_{n}\right)\right) \rightarrow H^{\star}\left(B O_{n+}\right) \rightarrow H^{\star}\left(B O_{n-1+}\right) \rightarrow H^{\star+1}\left(\operatorname{Thom}\left(B O_{n}\right)\right) \rightarrow \ldots
$$

Using the Thom isomorphism $H^{\star}\left(B O_{n+}\right) \stackrel{\cong}{\rightarrow} H^{\star+\omega_{n}}\left(\operatorname{Thom}\left(B O_{n}\right)\right)$ we get the long 
exact sequence

$$
\ldots \rightarrow H^{\star}\left(B O_{n+}\right) \stackrel{f_{n}^{*}}{\longrightarrow} H^{\star+\omega_{n}}\left(B O_{n+}\right) \stackrel{g_{n}^{*}}{\longrightarrow} H^{\star+\omega_{n}}\left(B O_{n-1+}\right) \stackrel{h_{n}^{*}}{\longrightarrow} H^{\star+1}\left(B O_{n+}\right) \rightarrow \ldots
$$

Notice that $f_{n}^{*}$ is multiplication by some nonzero class $w_{n}$. By induction, $H^{\star}\left(B O_{n-1+}\right)$ $=H^{\star}\left[w_{1}, \ldots, w_{n-1}\right]$. Since $B O_{n}$ is cellular, we have that $H^{p+q \alpha}\left(B O_{n+}\right)=0$ for $q<0$. It is also clear that the map $f_{n}^{*}$ is injective on $\mathbb{Z} / 2 \cong H^{0}\left(B O_{n+}\right)$. We can start with the case $n=0$ by identifying $B O_{0}$ with $\left|B\left(*, O_{1}, Q_{0}\right)\right|$ which is contractible. Therefore, we have that $h_{n}^{*}\left(w_{i}\right)=0$ for $i=0, \ldots, n-1$. It follows that each of the $w_{i}$ can be uniquely lifted to $H^{\star}\left(B O_{n+}\right)$. Moreover, since $h_{n}^{*}\left(w_{i}\right)=0$ for $i=0, \ldots, n-1$, it follows that $h_{n}^{*}=0$. Thus, the long exact exact sequence splits and we get the short exact sequence

$$
0 \rightarrow H^{\star}\left(B O_{n}\right) \stackrel{f_{n}^{*}}{\longrightarrow} H^{\star+\omega_{n}}\left(B O_{n}\right) \stackrel{g_{n}^{*}}{\longrightarrow} H^{\star+\omega_{n}}\left(B O_{n-1}\right) \rightarrow 0 .
$$

The key point is that $f_{n}^{*}$ is multiplication by the cohomology class $w_{n} \in H^{\omega_{n}}\left(B O_{n}\right)$. In other words $f_{n}^{*}=\smile w_{n}$.

From this the claim follows. We have

$$
H^{\star}\left(B O_{n}\right) \cong H^{\star}\left[w_{1}, \ldots, w_{n-1}\right] \oplus H^{\star}\left[w_{1}, \ldots, w_{n-1}\right] \smile w_{n} \cong H^{\star}\left[w_{1}, \ldots, w_{n}\right]
$$

If we define $B O:=\operatorname{colim}_{j>0}\left(B O_{j} \subset B O_{j+1}\right)$, then $H^{\star}(B O)=\lim _{\longleftarrow} H^{\star}\left(B O_{n+}\right)$.

A quick word is in order. We have a Thom isomorphism in (co)homology. I have computed the cohomology of $\mathrm{BO}_{n}$, but their is a motivic universal coefficient theorem, and so the (co)homology are essentially the same and their is a duality between the (co)homology classes. Motivically, this is not always the case. However, MGLO $\wedge H \mathbb{Z} / 2$ is a wedge sum of suspensions of $H \mathbb{Z} / 2$ of dimensions $p+q \alpha$ with 
$p \geq q$ and so we can show that the (co)homology classes are dual to one another [Hoy15]. This gives us the following theorem.

Theorem 2.12. There are a unique set of classes $w_{1}, w_{2}, \ldots, w_{n}$ belonging to motivic $\mathbb{Z} / 2$ homology for which,

$$
H_{\star}\left(B O_{n+}\right) \cong H^{\star}\left[w_{1}, \ldots, w_{n}\right]
$$

Here $\operatorname{deg}\left(w_{2 i}\right)=i+i \alpha$ and $\operatorname{deg}\left(w_{2 i+1}\right)=i+1+i \alpha$.

Since $H \mathbb{Z} / 2$ is an $\mathrm{E}_{\infty}$ ring spectrum, we have a universal coefficients theorem. Therefore, the $H \mathbb{Z} / 2$ cohomology classes in MGLO give dual homology classes in the coefficients of $\mathrm{MGLO} \wedge H \mathbb{Z} / 2$, and so we have that $H_{\star}(\mathrm{MGLO})$ is a free polynomial ring over generators $u_{k}$ with $\operatorname{deg}\left(u_{2 i}\right)=i+i \alpha$ and $\operatorname{deg}\left(u_{2 i+1}\right)=i+1+i \alpha$.

We now take a slight detour to discuss the Motivic Steenrod algebra.

\subsubsection{Dual Motivic Steenrod Algebra}

We can define the Dual Motivic Steenrod Algebra $\mathcal{A}_{M o t}^{\vee}$ to be $H \mathbb{Z} / 2 \wedge H \mathbb{Z} / 2$. As an $H_{\star}$ algebra, the coefficients of $\mathcal{A}_{M o t}^{\vee}$ are given by

$$
H_{\star}\left[\tau_{i}, \xi_{i+1}\right]_{i \geq 0} /\left(\tau_{i}^{2}-\tau \xi_{i+1}-\rho \tau_{i+1}-\rho \tau_{0} \xi_{i+1}\right)
$$

where $\left|\xi_{i+1}\right|=\left(2^{i+1}-1\right)(1+\alpha)$ and $\left|\tau_{i}\right|=\left(2^{i}-1\right)(1+\alpha)+1$. Let $\xi\left(r_{1}, r_{2}, \ldots, r_{n}\right):=$ $\xi^{r_{1}} \xi^{r_{2}} \ldots \xi^{r_{n}}$ for $r_{i} \in \mathbb{Z}^{\geq 0}$ and $\tau\left(i_{0}, i_{1}, \ldots, i_{m}\right):=\tau_{i_{0}}^{\epsilon_{0}} \tau_{i_{1}}^{\epsilon_{1}} \ldots \tau_{i_{m}}^{\epsilon_{m}}$ for $0 \leq i_{0}<i_{1}<\ldots<i_{m}$ and $\epsilon_{j} \in\{0,1\}$. It is clear from eq. (2.8) that a basis for $\mathcal{A}_{\text {Mot }}^{\vee}$ is given by products of the form $\xi\left(r_{1}, r_{2}, \ldots, r_{n}\right) \tau\left(i_{0}, i_{1}, \ldots, i_{m}\right)$.

By comparing the $H_{\star}$ module basis for the coefficients of MGLO $\wedge H$ and $\mathcal{A}_{M o t}^{\vee}$, we see that $\mathrm{MGLO} \wedge H$ is a wedge sum of suspensions of $\mathcal{A}_{M o t}^{\vee}$. Consider the submodule $\mathcal{M}$ of $H_{\star}(\mathrm{MGLO})$ obtained by deleting all generators of degree $\left(\xi_{i+1}\right)$ and squaring all 
generators of degree $\left(\tau_{i}\right)$. Let $\mathfrak{M}$ be an $H_{\star}$ module basis for this submodule. Then,

$$
\mathrm{MGLO} \wedge H \mathbb{Z} / 2 \simeq \bigvee_{m_{i} \in \mathfrak{M}} \Sigma^{\left|m_{i}\right|} \mathcal{A}_{M o t}^{\vee}
$$

\subsection{2 $2=\eta=0$ in $\mathrm{MGLO}_{\star}$}

Consider the stable cofibration induced by multiplication by 2 ,

$$
S^{0} \stackrel{2}{\rightarrow} S^{0} \rightarrow \mathrm{M}(2) .
$$

The cofiber $\mathrm{M}(2)$ is called the mod 2 Moore spectrum, and $H \mathbb{Z} \wedge \mathrm{M}(2) \simeq H \mathbb{Z} / 2$. Recall that classically $2=0$ in the coefficients of MO. The analogous statement will be shown to be true for MGLO.

Consider the Hopf map given by the projection $h: \mathbb{A}^{2} \backslash 0 \rightarrow \mathbb{P}^{1}$. Recall that $\mathbb{A}^{2} \backslash 0 \simeq S^{1+2 \alpha}$ and that $\mathbb{P}^{1} \simeq S^{1+\alpha}$. It follows that $h$ induces a stable map $\eta$ : $\Sigma^{\alpha} S^{0} \rightarrow S^{0}$. We denote the cokernel of this map by $S^{0} / \eta$. For a general spectrum E, we denote the cokernel of the map $\eta \wedge \mathrm{E}$ by $\mathrm{E} / \eta$.

Let $\mathrm{E}$ denote a cellular spectrum. We will say that $\mathrm{E}$ is $k$-connected if $\pi_{n+* \alpha}(\mathrm{E})=0$ for $n<k$. Analogously to the topological case, one can easily show that $\pi_{n+* \alpha}(\mathbb{Z} / 2) \cong$ $\pi_{n+1+* \alpha}\left(B C_{2}\right)$. Notice that $\pi_{0}(\mathbb{Z} / 2) \cong \mathbb{Z} / 2$.

Proposition 2.13. $2=0$ in the coefficients of MGLO.

The unit map $S^{0} \rightarrow$ MGLO can be decomposed as

$$
S^{0} \rightarrow \Sigma^{-1} B C_{2} \rightarrow \Sigma^{-1} \operatorname{Thom}\left(B C_{2}\right) \rightarrow \text { MGLO }
$$

Notice that $\mathbb{Z} / 2 \cong \pi_{1}(B \mathbb{Z} / 2) \cong \pi_{0}\left(\Sigma^{-1} B \mathbb{Z} / 2\right)$. Since the unit map of MGLO factors through the map representing the generator of $\pi_{0}\left(\Sigma^{-1} B C_{2}\right)$, it follows that $2=0$.

Proposition 2.14. $\eta=0$ in the coefficients of MGLO. 
Proof. It is well known that $\eta=0$ in the coefficients of MGL. Therefore, it will be enough to produce a map from MGL to MGLO. We accomplish this by producing a surjective map $G L_{n} \rightarrow O_{2 n}$. This map is given by $A \mapsto A \oplus\left(A^{T_{b}}\right)^{-1}$. This in turn induces a map $\mathrm{MGL} \rightarrow \mathrm{MGLO}$ as desired.

\subsubsection{Comodule structure of MGLO}

If $\mathrm{E}$ is a motivic spectrum, and $H$ denotes the mod 2 Moore spectrum, then $H_{\star}(\mathrm{E})$ has the structure of a left comodule over $\mathcal{A}_{\star}$. In particular, we can apply this to $\mathrm{E}=\mathrm{MGLO}$, giving us a coproduct:

$$
H_{\star}(\mathrm{MGLO}) \stackrel{\Delta}{\rightarrow} \mathcal{A}_{\star} \otimes_{H_{\star}} H_{\star}(\mathrm{MGLO}) .
$$

If we were to follow the classical argument, we would want to show that there exists a projection map $H_{\star}(\mathrm{MGLO}) \stackrel{\pi}{\rightarrow} C$, for some $H_{\star}$-module $C$, such that

$$
H_{\star}(\mathrm{MGLO}) \stackrel{\Delta}{\rightarrow} \mathcal{A}_{\star} \otimes_{H_{\star}} H_{\star}(\mathrm{MGLO}) \stackrel{1 \times \pi}{\longrightarrow} \mathcal{A}_{\star} \otimes_{H_{\star}} C
$$

is an isomorphism of left $\mathcal{A}_{\star}$-comodule algebras. Unfortunately this cannot be true. Notice that there is only one element of degree 1 in $H_{\star}(\mathrm{MGLO})$. Call this element $u_{1}$. Since $H_{\star}(\mathrm{MGLO})$ is an $H_{\star}$-polynomial algebra on infinitely many generators, one of which is $u_{1}$, it follows that $u_{1}^{2}$ must represent the single $H_{\star}$-module basis element of $H_{\star}(\mathrm{MGLO})$ in degree 2 . However, as an $H_{\star}$-module, $\mathcal{A}_{\star}$ has no basis element of degree 2. It follows that $(1 \times \pi) \circ \Delta\left(u_{1}\right)=\tau_{0}$ and $(1 \times \pi) \circ \Delta\left(u_{1}^{2}\right) \in C$. However, this contradicts the formula $(1 \times \pi) \circ \Delta\left(u_{1}^{2}\right)=\left((1 \times \pi) \circ \Delta\left(u_{1}\right)\right)\left((1 \times \pi) \circ \Delta\left(u_{1}\right)\right)$.

One notices that while $H_{\star}(\mathrm{MGLO})$ is not equal to $\mathcal{A}_{\star} \otimes_{H_{\star}} C$ as an $H_{\star}$-algebra, it is as an $H_{\star}$-module. This is accomplished by simply comparing the $H_{\star}$-module basis of $H_{\star}(\mathrm{MGLO})$ to the $H_{\star}$-module basis of $\mathcal{A}_{\star}$, and then observing that as an 
$H_{\star}$-module, $H_{\star}(\mathrm{MGLO})$ is a direct sum of $\mathcal{A}_{\star}$. Recalling that $\mathcal{A} \simeq H \wedge H$, it follows that $H \wedge \mathrm{MGLO} \simeq H \wedge\left(\bigvee_{i \in \mathcal{S}} \Sigma^{a_{i}} H\right)$. One then only has to construct a map between MGLO and $\bigvee_{i \in \mathcal{S}} \Sigma^{a_{i}} H$ which is an isomorphism on $H \mathbb{Z} / 2$ homology. The point is that for each $i \in \mathcal{S}$, there exists a cohomology basis element $a_{i} \in H^{a_{i}}$ (MGLO) representing the map MGLO $\rightarrow \Sigma^{a_{i}} H$. Piecing these cohomology classes together, this gives a map MGLO $\stackrel{f}{\rightarrow} \bigvee_{i \in \mathcal{S}} \Sigma^{a_{i}} H$. Since (co)homology classes of MGLO are dual to one another, it follows that the map $f$ induces an equivalence on homology.

\subsubsection{Applying the Motivic Hurewicz Theorem}

We will use a modified version of the Motivic Hurewicz Theorem of [Bac15].

We recall what it means to be $(n-1)$-connected in the motivic sense.

Definition 2.15. We say that a motivic spectrum $\mathrm{E}$ is $(n-1)$-connected if $\pi_{k+* \alpha}(\mathrm{E})=$ 0 whenever $0<k<n$. We also require that $\pi_{k+m \alpha}(\mathrm{E})=0$ for all but at most finitely many $m \in \mathbb{Z}$.

Theorem 2.16. Let $\mathrm{k}$ have characteristic 0 , and suppose that $\mathrm{E}$ is an $(n-1)$-connected cellular stable motivic spectrum for which 2 and $\eta$ are 0 . Then

$$
H_{n+* \alpha}(\mathrm{E} ; \mathbb{Z} / 2) \cong \pi_{n+* \alpha}(\mathrm{E})
$$

Consider the basis elements $v_{i} \in \mathfrak{M} \subset H_{\star}(\mathrm{MGLO})$. Then each of the $v_{i}$ is dual to a cohomology class $c_{i} \in H^{\star}(\mathrm{MGLO})$, and so there exists a map

$$
\operatorname{MGLO} \stackrel{f}{\rightarrow} \bigvee_{m_{i} \in \mathfrak{M}} \Sigma^{\left|m_{i}\right|} H \mathbb{Z} / 2
$$

which induces an equivalence on homology. Taking the cofiber of the map $f$ we obtain a cofibration

$$
\operatorname{MGLO} \stackrel{f}{\rightarrow} \bigvee_{m_{i} \in \mathfrak{M}} \Sigma^{\left|m_{i}\right|} H \mathbb{Z} / 2 \rightarrow \mathrm{F}
$$


The idea is that we know that $\mathrm{F}$ is cellular, and the coefficients of $\mathrm{F} \wedge H \mathbb{Z} / 2$ are 0 by construction. Since 2 and $\eta$ are 0 in $\bigvee_{m_{i} \in \mathfrak{M}} \Sigma^{\left|m_{i}\right|} H \mathbb{Z} / 2$, it follows that $2^{2}$ and $\eta^{2}$ are 0 in $\mathrm{F}$ and so the Motivic Hurewicz Theorem combined with the Nakayama lemma implies that $\mathrm{F}=0$ and so $f$ is an equivalence.

\subsection{5 $\mathrm{MGLO}_{\star}$ and a comparison with $\mathrm{MO}_{*}$}

Combining everything, we have that

Theorem 2.17. As an $H_{\star}$ algebra,

$$
\mathrm{MGLO}_{\star} \cong H_{\star}\left[u_{n+n \alpha}, u_{n+1+n \alpha}, u_{\left(2^{i}-1\right)(1+\alpha)+2} \mid n, i \in \mathbb{Z}^{\geq 0}, n \neq 2^{i}-1\right] .
$$

Let $t^{\mathbb{C}}$ denote the complex topological realization functor. Then

$$
\begin{array}{r}
t^{\mathbb{C}}\left(S^{1}\right)=S^{1}, \\
t^{\mathbb{C}}\left(S^{\alpha}\right)=S^{1}, \\
t^{\mathbb{C}}\left(H \mathbb{Z} / 2_{M o t}\right)=H \mathbb{Z} / 2 .
\end{array}
$$

From this it follows that $t^{\mathbb{C}}(\mathrm{MGLO})=\mathrm{MO}$. Over $\mathrm{k}=\mathbb{C}$, we have that

$$
\begin{array}{r}
\mathrm{MGLO}_{\star}=H \mathbb{Z} / 2_{M o t \star}\left[x_{2}, x_{2+2 \alpha}, x_{3+2 \alpha}, u_{4+2 \alpha}, u_{4+4 \alpha}, u_{5+4 \alpha}, x_{5+5 \alpha}, \ldots\right] \\
=\mathbb{Z} / 2[\theta]\left[u_{2}, u_{2+2 \alpha}, x_{3+2 \alpha}, u_{4+2 \alpha}, u_{4+4 \alpha}, u_{5+4 \alpha}, u_{5+5 \alpha}, \ldots\right] \\
=\mathbb{Z} / 2\left[\theta, u_{2}, u_{2+2 \alpha}, u_{3+2 \alpha}, u_{4+2 \alpha}, u_{4+4 \alpha}, u_{5+4 \alpha}, u_{5+5 \alpha}, \ldots\right] .
\end{array}
$$

Recall that

$$
\mathrm{MO}_{*}=\mathbb{Z} / 2\left[a_{2}, a_{4}, a_{5}, a_{6}, a_{8}, a_{9}, a_{10}, \ldots\right]
$$

So the generators of $\mathrm{MO}_{*}$ correspond to generators in $\mathrm{MGLO}_{\star}$ twisted by powers of $\theta$. 


\subsubsection{The topological realization of MGLO over $\mathbb{R}$}

The following results about topological realization functors can be found in [HK11, HVØ16]. The application discussed herein is an observation of the current author. There is a topological realization functor from the motivic stable homotopy category to the $C_{2}$-equivariant stable homotopy category, and from the $C_{2}$-equivariant motivic stable homotopy category to the $C_{2} \times C_{2}$-equivariant stable homotopy category. Let us start off with the realization functor $t_{C_{2}}^{\mathbb{R}}$ which lands in $\mathrm{SH}_{C_{2}}$. At the level of schemes, the functor $t_{C_{2}}^{\mathbb{R}}$ sends real algebraic varieties to the $\mathbb{C}$ points of $X$, which we denote $X(\mathbb{C})$. This gives a complex manifold, and since $X$ is a real algebraic variety, we have a group action $\operatorname{Gal}(\mathbb{C} / \mathbb{R}) \cong C_{2}$. This gives us the desired functor. On the other hand, the real algebraic variety $X$ may have already been equipped with some sort of $C_{2}$ action. We could combine these two $C_{2}$ actions to get a functor $t_{C_{2} \times C_{2}}^{\mathbb{R}}$ landing in the $C_{2} \times C_{2}$ stable homotopy category.

In particular, the functor $t_{C_{2} \times C_{2}}^{\mathbb{R}}$ sends $M G L R$ to $M R_{\mathbb{Z} / 2}$. We can use this to figure out where $t_{C_{2} \times C_{2}}^{\mathbb{R}}$ sends MGLO. $\mathrm{MR}_{\mathbb{Z} / 2}$ is a 4-graded ring spectrum, and the grading is $1, \alpha, \sigma, \sigma \alpha$.

We denote the nonzero elements $(1,0),(0,1)$, and $(1,1)$ of $C_{2} \times C_{2}$ by $g_{\alpha}, g_{\sigma}$, and $g_{\sigma \alpha}$ respectively. We write $\alpha$ for the $C_{2} \times C_{2}$ representation which is defined by letting $g_{\alpha}$ act by -1 and $g_{\sigma}$ act by the identity. We write $\sigma$ for the $C_{2} \times C_{2}$ representation which is defined by letting $g_{\sigma}$ act by -1 and $g_{\alpha}$ act by the identity. We write $\sigma \alpha$ for the $C_{2} \times C_{2}$ representation $g_{\sigma \alpha}=g_{\sigma} \otimes g_{\alpha}$ which is defined by letting $g_{\sigma}$ and $g_{\alpha}$ act by -1 .

The effect of the topological realization functor on the spheres is as follows. 
Lemma 2.18. We have

$$
\begin{array}{r}
t_{C_{2} \times C_{2}}^{\mathbb{R}}\left(S^{1}\right) \simeq S^{1}, t_{C_{2} \times C_{2}}^{\mathbb{R}}\left(S^{\sigma}\right) \simeq S^{\sigma}, \\
t_{C_{2} \times C_{2}}^{\mathbb{R}}\left(S^{\alpha}\right) \simeq S^{\alpha}, t_{C_{2} \times C_{2}}^{\mathbb{R}}\left(S^{\sigma \alpha}\right) \simeq S^{\sigma \alpha} .
\end{array}
$$

Now, by the periodicities of $\mathrm{MR}_{\mathbb{Z} / 2}$, it follows that

$$
\begin{array}{r}
t_{C_{2} \times C_{2}}^{\mathbb{R}}(\mathrm{MGLO})=t_{C_{2} \times C_{2}}^{\mathbb{R}}\left(\Phi_{e t}^{C_{2}}(\mathrm{MGLR})\right)=\left(S^{\infty \alpha+\infty \sigma} \wedge t_{C_{2} \times C_{2}}^{\mathbb{R}}(\mathrm{MGLR})\right)^{C_{2}\left\{g_{\sigma \alpha}\right\}} \\
=\left(S^{\infty \alpha+\infty \sigma} \wedge \mathrm{MR}_{\mathbb{Z} / 2}\right)^{C_{2}\left\{g_{\sigma \alpha}\right\}}
\end{array}
$$

From this, it follows by [HK01, page 9] that

$$
t_{C_{2} \times C_{2}}^{\mathbb{R}}(\mathrm{MGLO})=\bigvee_{n \in \mathbb{Z}} \Sigma^{n(1+\sigma \alpha)} B \mathbb{U}_{+} \wedge \mathrm{MR}
$$

where $\mathbb{U}$ is the infinite unitary group with $C_{2}$-action given by complex conjugation. 


\section{CHAPTER III}

\section{A motivic analogue of MSO}

Recall that the classical oriented cobordism spectrum MSO is closely related to MO. Similarly to MO, the spectrum MSO can be constructed from the thom spaces of the classifying spaces of $S O_{n}$, which we denote by $B S O_{n}$. Recall that the group $S O_{n}$ is defined as $\left\{A \in O_{n} \mid \operatorname{det}(A)=1\right\}$.

Although many results found in this chapter can be generalized to more general fields, many of the proofs will rely on the coefficients of the motivic $\mathbb{Z} / p$ cohomology of the $\bmod p$ Eilenberg-Maclane spectrum being equal to $\mathbb{Z} / 2[\tau]$, where $\tau$ denotes the tate twist of degree $\alpha-1$. Therefore, for the entirety of Chapter 3 , the reader should always assume that $\sqrt{-1} \in \mathrm{k}$, and that $\mathrm{k}$ is a field of characteristic 0 .

\subsection{Computing the coefficients of MSLO}

Having constructed a motivic analogue of MO, it became apparent that it would be possible to construct a motivic analogue of MSO by mimicking the construction of MGLO. The simple observation is that we can again consider the quadratic form,

$$
q\left(x_{1}, x_{2}, \ldots, x_{2 n}\right)=x_{1} x_{2}+x_{3} x_{4}+\ldots+x_{2 n-1} x_{2 n} .
$$


To this we can associate a unique orthogonal group $O_{2 n}$. Since the determinant function is algebraic, we can define the $2 n$-dimensional special orthogonal groups as,

$$
S O_{2 n}:=\left\{A \in O_{2 n} \mid \operatorname{det} A=1\right\}
$$

Again, for $n \geq 1$ we get a transitive group action of $\mathrm{SO}_{2 n}$ on

$$
Q_{2 n-1}:=\mathbb{V}\left(x \in \mathrm{k}^{2 n} \mid q(x)-1\right) \simeq S^{n-1+n \alpha}
$$

Letting $x^{0}=\langle 1,1.0, \ldots, 0\rangle$, the stabilizer of $x^{0}$ with respect to the group action of $S O_{2 n}$ on $Q_{n-1}$ is defined to be $S O_{2 n-1}$. One easily sees that this is exactly equal to $\left\{A \in O_{2 n-1} \mid \operatorname{det}(A)=1\right\}$. Defining as before

$$
Q_{2 n-2}:=\mathbb{V}\left(x \in \mathrm{k}^{2 n} \mid q(x)+1, x_{1}+x_{2}\right) \simeq S^{n-1+(n-1) \alpha},
$$

we get a group action of $S O_{2 n-1}$ on $Q_{2 n-2}$. This action is transitive, and defining $y^{0} \in \mathrm{k}^{2 n}$ to be $\langle 1,-1,0, \ldots, 0\rangle$, we can show that the stabilizer of $y^{0}$ is $S O_{2 n-2}$.

In the lower dimensional cases, we note that $S O_{2} \simeq \mathbb{G}_{m}$, and $S O_{1} \simeq *$. The later equivalence is obvious. For the former, we have to do a bit of work.

Proposition 3.1. $\mathrm{SO}_{2} \simeq \mathbb{G}_{m}$.

Proof. We consider the symmetric bilinear form $b\left(\left(x_{1}, x_{2}\right),\left(y_{1}, y_{2}\right)\right)$ to see how $A$ is related to $A^{T}$. Recall that $A^{T}$ is defined to be the unique matrix $A \in G L_{2}(\mathrm{k})$ for which $b(A x, y)=b\left(x, A^{T} y\right)$. We write

$$
A=\left[\begin{array}{ll}
a & b \\
c & d
\end{array}\right], A^{T}=\left[\begin{array}{ll}
a^{\prime} & b^{\prime} \\
c^{\prime} & d^{\prime}
\end{array}\right], x=\left\langle x_{1}, x_{2}\right\rangle, y=\left\langle y_{1}, y_{2}\right\rangle
$$


Recall that $b(x, y)=x_{1} y_{2}+x_{2} y_{1}$. Therefore,

$$
b(A x, y)=a x_{1} y_{2}+b x_{2} y_{2}+c x_{1} y_{1}+d x_{2} y_{1}
$$

and

$$
b\left(x, A^{T} y\right)=c^{\prime} x_{1} y_{1}+d^{\prime} x_{1} y_{2}+a^{\prime} x_{2} y_{1}+b^{\prime} x_{2} y_{2}
$$

Comparing, we see that

$$
A^{T}=\left[\begin{array}{ll}
d & b \\
c & a
\end{array}\right]
$$

Now notice that we have the further relations $\operatorname{det}(A)=1$ and $A A^{T}=I$. Explicitly multiplying the matrices, we see that

$$
A A^{T}=\left[\begin{array}{cc}
a d+b c & 2 a b \\
2 c d & a d+b c
\end{array}\right] \text {. }
$$

Since $\operatorname{det}(A)=a d-b c=1$, we have that $a d+b c=(a d-b c)+2 b c=1+2 b c$. Therefore, we get the relations $2 b c=2 a b=2 c d=0$. It follows, from these relations alone, that either $a=c=0, b=c=0$, or $b=d=0$. But we also have the relation $a d-b c=1$. Therefore, it must be the case that $b=c=0$. Therefore,

$$
S O_{2}=\left\{(a, b, c, d) \in \mathrm{k}^{4} \mid b=c=0, a d=1\right\} \simeq\left\{(v, w) \in \mathrm{k}^{2} \mid v w=1\right\} \simeq \mathbb{G}_{m}
$$

Using a two sided bar construction as before, we have

$$
\left|B\left(S O_{n}, S O_{n-1}, *\right)\right| \simeq Q_{n-1}
$$


Moreover, we are able to show that

$$
\left|B\left(*, S O_{n}, Q_{n-1}\right)\right| \simeq B S O_{n-1} .
$$

We are able to define the thom spaces for prespectrum of MSLO in the same way as before as the homotopy cofiber of

$$
\mathrm{BSO}_{n-1_{+}} \rightarrow \mathrm{BSO}_{n+}
$$

Notice that in particular we have,

Lemma 3.2. $\mathbb{P}^{\infty} \simeq B \mathbb{G}_{m} \simeq B S O_{2} \simeq \operatorname{Thom}\left(B S O_{2}\right)$.

Proof. Since $S O_{1} \simeq *$, we have $B S O_{1} \simeq *$. By definition of Thom $\left(B S O_{2}\right)$, the statement follows.

\subsubsection{Calculating the $\mathbb{Z} / 2$ cohomology of MSLO}

The goal of this section is to calculate the motivic $\mathbb{Z} / 2$ cohomology of MSLO. To do this, we first note that $O_{n}$ acts on the unit sphere $S^{0} \simeq\{ \pm 1\}$ by $A \cdot g \mapsto(\operatorname{det}(A)) g$ for $A \in O_{n}, g \in\{ \pm 1\}$. This action is easily seen to be transitive, and the stabilizer of $1 \in S^{0}$ is $\left\{A \in O_{n} \mid \operatorname{det}(A)=1\right\}=S O_{n}$. It follows that $\left|B\left(*, O_{n}, S^{0}\right)\right| \simeq B S O_{n}$. As before, we get a thom isomorphism

$$
H^{\star}\left(B O_{n+}\right) \cong \widetilde{H}^{\star+1}\left(B O_{n} / B S O_{n}\right)
$$

We can use this to get a Gysin sequence. We consider the long exact sequence

$$
\ldots \rightarrow H^{\star}\left(B O_{n} / B S O_{n}\right) \rightarrow H^{\star}\left(B O_{n+}\right) \rightarrow H^{\star}\left(B S O_{n+}\right) \rightarrow H^{\star+1}\left(B O_{n} / B S O_{n}\right) \rightarrow \ldots
$$


Substituting in the thom isomorphism gives us,

$$
\begin{array}{r}
\ldots \rightarrow H^{\star-1}\left(B O_{n+}\right) \rightarrow H^{\star}\left(B O_{n+}\right) \rightarrow H^{\star}\left(B S O_{n+}\right) \rightarrow H^{\star}\left(B O_{n+}\right) \rightarrow \\
H^{\star+1}\left(B O_{n+}\right) \rightarrow \ldots
\end{array}
$$

Proposition 3.3. There exists a surjective map,

$$
H^{\star}\left(B O_{n+}\right) \rightarrow H^{\star}\left(B S O_{n+}\right)
$$

with kernel generated by $w_{1}$ as an $H^{\star}$ module. Hence, $H^{\star}\left(B S O_{n+}\right) \cong H^{\star}\left[w_{2}, w_{3}, \ldots, w_{n}\right]$ with $\left|w_{2 i}\right|=i+i \alpha$, and $\left|w_{2 i+1}\right|=i+1+i \alpha$.

Proof. Form $x \in H^{1}\left(B O_{n_{+}}\right)$as the composition of the thom class $u \in H^{1}\left(B O_{n} / B S O_{n}\right)$ with the homotopy cofiber of the the map

$$
\mathrm{BSO}_{n+} \rightarrow \mathrm{BO}_{n+}
$$

This gives a nonzero class $x \in H^{1}\left(B O_{n_{+}}\right)$. Since there is only one nonzero class $H^{\star}\left(B O_{n_{+}}\right)$of degree 1 , it is clear that $x$ is the same class as $w_{1} \in H^{1}\left(B O_{n+}\right)$ from Theorem theorem 2.11.

Thus, we can write

$$
\ldots \rightarrow H^{\star}\left(B O_{n+}\right) \rightarrow H^{\star}\left(B S O_{n+}\right) \rightarrow H^{\star}\left(B O_{n+}\right) \stackrel{\smile w_{1}}{\longrightarrow} H^{\star+1}\left(B O_{n+}\right) \rightarrow \ldots
$$

Since $H^{\star}\left(B O_{n+}\right)=H^{\star}\left[w_{1}, \ldots, w_{n}\right]$, the map $\smile w_{1}$ is injective in all dimensions, and so the Gysin sequence breaks up into short exact sequences

$$
0 \rightarrow H^{r+s \alpha-1}\left(B O_{n+}\right) \stackrel{-w_{1}}{\longrightarrow} H^{r+s \alpha}\left(B O_{n+}\right) \rightarrow H^{r+s \alpha}\left(B S O_{n+}\right) \rightarrow 0 .
$$

The conclusion follows. 


\subsection{2 $\quad H_{\star}(H \mathbb{Z})$ comodule structure of MSLO}

Classically, Pengelley gives a description of the $H \mathbb{Z} / 2$-homology of MSO [Pen82] to be $H_{\star}(H \mathbb{Z}) \otimes_{\mathbb{F}_{2}} \mathbb{F}_{2}\left[x_{k} \mid k \neq 2, k \neq 2^{i}-1\right]$. We can give a similar description of MSLO as an $H_{\star}$-module, although this will not be the approach we will end up taking in proving our main results. Nevertheless, the description is interesting. One realizes that since MSLO is cellular, $H \mathbb{Z} / 2 \wedge$ MSLO must be equivalent to a wedge sum of suspensions of motivic $H \mathbb{Z} / 2$. Moreover, $H \mathbb{Z}$ is known to be cellular, which means that $H \mathbb{Z} / 2 \wedge H \mathbb{Z}$ must be a wedge sum of suspensions of motivic $H \mathbb{Z} / 2$. One then notices by inspection that $H \wedge \mathrm{MSLO}$ is equivalent to a wedge sum of suspensions of $H \wedge H \mathbb{Z}$. While we cannot give a nice algebra description of $H \wedge$ MSLO in this manner, we may give a nice description of the $H_{\star}$-module structure of $H \wedge$ MSLO. Let $\pi_{\star}(\mathrm{MGLO})=H_{\star}\left[u_{k} \mid k \neq 2^{i}-1\right]$. Then, as an $H_{\star}$-module, we have that

$$
H_{\star}(\mathrm{MSLO}) \cong H_{\star}(H \mathbb{Z}) \otimes_{H_{\star}} H_{\star}\left[u_{k} \mid k \neq 2, k \neq 2^{i}-1\right] .
$$

\subsubsection{Calculating the $\mathbb{Z} / p$ cohomology of MSLO for $p$ an odd prime}

Definition 3.4. The Euler class $x_{n} \in H^{\omega_{n}}\left(B S O_{n+}\right)$ is defined to be the composition of the thom class $c \in H^{\omega_{n}}\left(\operatorname{Thom}\left(B S O_{n}\right)\right)$ with the homotopy cofiber $f$ of

$$
B S O_{n-1_{+}} \rightarrow B S O_{n+} \stackrel{f}{\rightarrow} \text { Thom }\left(B S O_{n}\right) .
$$

Theorem 3.5. $H^{\star}\left(B S O_{n+} ; \mathbb{Z} / p\right)$ is the polynomial ring $H \mathbb{Z} / p^{\star}\left[x_{1}^{2}, \ldots, x_{k}^{2}\right]$ for $n=$ $2 k+1$ and $H \mathbb{Z} / p^{\star}\left[x_{1}^{2}, \ldots, x_{k-1}^{2}, x_{k}\right]$ for $n=2 k$.

Proof. The sphere bundle $S(n-1) \rightarrow B S O_{n-1} \rightarrow B S O_{n}$ induces a Gysin sequence 
with $\mathbb{Z} / p$ coefficients.

$$
\begin{array}{r}
\ldots \rightarrow H^{i}\left(B S O_{n+}\right) \stackrel{x_{n}}{\longrightarrow} H^{i+\omega_{n}}\left(B S O_{n+}\right) \stackrel{g_{n}^{*}}{\longrightarrow} H^{i+\omega_{n}}\left(B S O_{n-1+}\right) \stackrel{h_{n}^{*}}{\longrightarrow} \\
H^{i+1}\left(B S O_{n+}\right) \rightarrow \ldots
\end{array}
$$

Now, if $n=2 k$, then by induction we have that $H^{\star}\left(B S O_{n-1_{+}}\right) \cong H^{\star}\left[x_{1}^{2}, \ldots, x_{k-1}^{2}\right]$. Recall that by [MVW11], $H \mathbb{Z} / p_{\star}^{m+n \alpha}\left(B O_{n+}=0\right.$ for $n<0$. Using the fact that $\smile x_{n}$ is an isomorphism on $H^{0}\left(B S O_{n_{+}}\right) \cong \mathbb{Z} / p$, we see that $h_{n}^{*}=0$ and so $g_{n}^{*}$ is surjective and the map breaks into short exact sequences. The proof then follows that of Theorem theorem 2.11.

If $n=2 k+1$, then $x_{n}$ is zero in $H^{\omega_{n}}\left(B S O_{n+}\right)$ since it has order 2. To see that $x_{n}$ has order 2 , we note that $x_{n}$ is the element corresponding to $x_{n} \smile x_{n}$ under the thom isomorphism. Therefore, $x_{n} \smile x_{n}=-x_{n} \smile x_{n}$ by the commutativity relation of the cup product. It follows that $\smile x_{n}=0$ and so the Gysin sequence splits into short exact sequences

$$
0 \rightarrow H^{i+\omega_{n}}\left(B S O_{n+}\right) \stackrel{g_{n}^{*}}{\rightarrow} H^{i+\omega_{n}}\left(B S O_{n-1_{+}}\right) \stackrel{h_{n}^{*}}{\rightarrow} H^{i+1}\left(B S O_{n+}\right) \rightarrow 0
$$

Therefore $g_{n}^{*}$ injects $H^{\star}\left(B S O_{n+}\right)$ as a subring of $H^{\star}\left(B S O_{n-1+}\right) \cong$ $H^{\star}\left[w_{1}^{2}, \ldots, w_{k-1}^{2}, w_{k}\right]$. The subring $\operatorname{Im}\left(g_{n}^{*}\right)$ contains $H^{\star}\left[x_{1}^{2}, \ldots, x_{k}^{2}\right]$, and we can show it equals this ring by comparing ranks in each dimension.

\subsubsection{Calculating the coefficients of $\mathrm{MSLO}_{p}$ for $p$ an odd prime}

Recall that the computation of MSO at an odd prime is more of less the same as the computation of complex cobordism MU. Similarly, the computation of MSLO will be no harder than the computation of MGL.

We denote the Milnor primitives by $Q_{i} \in \mathcal{A}^{\star},\left|Q_{i}\right|=p^{i}(1+\alpha)-\alpha$. Recall that if $p$ is odd, then the mod $p$ motivic cohomology of MSLO is generated by classes $x_{i}$ of 
degree $2(1+\alpha) i$ as a free $H^{\star}$-module.

The following proof is based off the proof of a similar result due to S. Borghesi [Bor03, Proposition 6].

Theorem 3.6. Let $p$ be an odd prime. The mod $p$ cohomology of MSLO takes the form

$$
H^{\star}(\mathrm{MSLO})=\left(\mathcal{A}^{\star} /\left(Q_{0}, Q_{1}, \ldots\right)\right)\left[m_{i} \mid i \neq p^{n}-1\right]
$$

as an $\mathcal{A}^{\star}$-module where $\left|m_{i}\right|=2 i(1+\alpha)$.

Proof. For $c$ a cohomology class of degree $p+q \alpha$, we define $\|c\|:=p-q$. We will call the number $\|c\|$ the invariance of the cohomology class $c$. Now note that the motivic steenrod algebra $\mathcal{A}^{\star}$ acts on the cohomology of MSLO. Let $Q_{i}$ denote the Milnor primitives in degree $2^{i}(1+\alpha)-\alpha$. Notice that $\left\|Q_{i}\right\|=1$. Recall that as an $H^{\star}$ module, the cohomology of MSLO has a basis in monomials whose invariance is equal to 0 . Call this basis $\mathfrak{M}$. Therefore, $\left\|Q_{i} c\right\|=1$ imples that $Q_{i} c=0$. The reason is because for and $x \in H^{\star},\|x\| \leq 0$. Putting this together, we have that if $m \in \mathfrak{M}$, and that $y$ is a basis element of $\mathcal{A}^{\star}$ as an $H^{\star}$ module, then the action of $y$ on $m$ sends $m$ to a sum of elements in $\mathfrak{M}$ with coefficients in $\mathbb{Z} / 2$. Now, since $Q_{i} c=0$ for all $c \in \mathfrak{M}$, it follows that the action of $\mathcal{A}^{\star}$ on $H^{\star}$ (MSLO) factors through $\mathcal{A}^{\star} /\left(Q_{0}, Q_{1}, \ldots\right)$. By discussion of $\mathcal{A}^{\star}$ on the cohomology of MSLO, it now follows that that the action produces an $H^{\star}$ linear map in which there is no interplay between the $H^{\star}$ coefficients. Therefore, any dependencies must be topologically induces. But topologically, there are no dependencies, and so the theorem is proved.

Corollary 3.7. Let $p$ be an odd prime. The mod $p$ cohomology of MSLO takes the form

$$
H^{\star}(\mathrm{MSLO})=H^{\star}(\mathrm{BPGL})\left[m_{i} \mid i \neq p^{n}-1\right]
$$

as an $\mathcal{A}^{\star}$-module where $\left|m_{i}\right|=2 i(1+\alpha)$. 
For the remainder of this subsection, we will be over the field $\mathrm{k}=\mathbb{C}$. By [Sta16], we know that over $\mathbb{C}$, the motivic $\mathbb{Z} / p$ cohomology of a point is equal to $\mathbb{Z} / p[\tau]$, where $|\tau|=\alpha-1$. Dually, the motivic $\mathbb{Z} / p$ homology of a point is equal to $\mathbb{Z} / p[\theta]$ where $|\theta|=1-\alpha$. Furthermore, we have that $\mathcal{A}_{\star} \cong \mathcal{A}_{\star}^{\text {top }} \otimes_{\mathbb{Z} / p} \mathbb{Z} / p[\theta]$.

Definition 3.8. Let $\mathcal{E}(n), 0 \leq n<\infty$, denote the qoutient Hopf algebroid

$$
\mathcal{E}(n):=\mathcal{A}_{\star} / /\left(\xi_{1}, \xi_{2}, \ldots, \tau_{n+1}, \tau_{n+2}, \ldots\right)=H_{\star}\left[\tau_{0}, \ldots, \tau_{n}\right] /\left(\tau_{i}^{2} \mid 0 \leq i \leq n\right) .
$$

If $n=\infty$, let

$$
\mathcal{E}(\infty):=\mathcal{A}_{\star} / /\left(\xi_{1}, \xi_{2}, \ldots\right)=H_{\star}\left[\tau_{0}, \tau_{1}, \ldots\right] /\left(\tau_{i}^{2} \mid 0 \leq i\right) .
$$

There is a way of switching between $\mathcal{A}^{*}$ structures on cohomology and $\mathcal{A}_{*}$ structures on homology. In our case we have the following.

Proposition 3.9. As an $\mathcal{A}_{\star}$-comodule algebra, $H_{*} \mathrm{BPGL}=\mathcal{A}_{*} \square_{\mathcal{E}(\infty)} H_{*}$.

Using a change of rings isomorphism, we have

$$
\operatorname{Ext}_{\mathcal{A}_{\star}}\left(H_{\star}, H_{\star}(\mathrm{BPGL})\right) \cong \operatorname{Ext}_{\mathcal{A}_{\star}}\left(H_{\star}, \mathcal{A}_{\star} \square \bar{E}(\infty) H_{\star}\right) \cong \operatorname{Ext}_{\mathcal{E}(\infty)}\left(H_{\star}, H_{\star}\right) .
$$

If we let $\mathcal{E}(\infty)^{\text {top }}$ and $H_{\star}^{\text {top }}$ denote the topological analogues of $\mathcal{E}(\infty)$ and $H_{\star}$ respectively, then it follows that over $\mathrm{k}=\mathbb{C}$,

$$
\operatorname{Ext}_{\mathcal{E}(\infty)}\left(H_{\star}, H_{\star}\right) \cong \operatorname{Ext}_{\mathcal{E}(\infty)^{\text {top }}}\left(H_{\star}^{\text {top }}, H_{\star}^{\text {top }}\right) \otimes_{\mathbb{Z} / p} \mathbb{Z} / p[\theta] .
$$

From here the proof proceeds classically, and so we have the following theorem.

Theorem 3.10. After completing at an odd prime $p$, the coefficients of MSLO are given by

$$
\pi_{\star}\left(\mathrm{MSLO}_{p}\right) \cong \mathbb{Z}_{(p)}\left[\theta, x_{1}, x_{2}, x_{3}, \ldots\right]
$$


where $\left|x_{i}\right|=2 i(1+\alpha)$.

\subsection{5 $H \mathbb{Z} / 2_{\star}$-algebra structure of $H_{\star}(H \mathbb{Z} ; \mathbb{Z} / 2)$.}

By [Voe03], the map

$$
\psi_{*}: \mathcal{A}_{\star} \rightarrow \mathcal{A}_{\star} \otimes_{H_{\star}} \mathcal{A}_{\star}
$$

is given by

$$
\begin{gathered}
\psi_{*}\left(\xi_{k}\right)=\sum_{i=0}^{k} \xi_{k-i}^{2^{i}} \otimes \xi_{i}, \\
\psi_{*}\left(\tau_{k}\right)=\sum_{i=0}^{k} \xi_{k-i}^{2^{i}} \otimes \tau_{i}+\tau_{k} \otimes 1 .
\end{gathered}
$$

As in [Mil58], we define the conjugate of $\xi_{i}$ and $\tau_{i}$ inductively as

$$
\begin{gathered}
\sum_{i=0}^{k} \xi_{k-i}^{2^{i}} \otimes c\left(\xi_{i}\right)=0, \\
\sum_{i=0}^{k} \xi_{k-i}^{2^{i}} \otimes c\left(\tau_{i}\right)+\tau_{k} \otimes 1=0
\end{gathered}
$$

respectively.

This gives us

$$
\begin{gathered}
c\left(\xi_{k}\right)=-\xi_{k}-c\left(\xi_{1}\right) \xi_{k-1}^{2} \ldots-c\left(\xi_{k-1}\right) \xi_{1}^{2^{k-1}} \\
c\left(\tau_{k}\right)=-\tau_{k}-c\left(\tau_{0}\right) \xi_{k}-c\left(\tau_{1}\right) \xi_{k-1}^{2}-\ldots-c\left(\tau_{k-1}\right) \xi_{1}^{2^{k-1}}
\end{gathered}
$$

respectively.

As in topology, motivically we have a cofibration

$$
H \mathbb{Z} \stackrel{2}{\rightarrow} H \mathbb{Z} \stackrel{\bmod 2}{\longrightarrow} H \mathbb{Z} / 2
$$


induced from the short exact sequence

$$
0 \rightarrow \mathbb{Z} \stackrel{2}{\rightarrow} \mathbb{Z} \stackrel{\bmod 2}{\longrightarrow} \mathbb{Z} / 2 \rightarrow 0
$$

Taking motivic $H \mathbb{Z} / 2$ homology of $H \mathbb{Z}$, we get a long exact sequence

$$
\ldots \rightarrow H^{\star}(H \mathbb{Z}) \stackrel{2}{\rightarrow} H^{\star}(H \mathbb{Z}) \stackrel{\bmod 2}{\longrightarrow} H^{\star}(H \mathbb{Z} / 2) \stackrel{\partial}{\rightarrow} \ldots
$$

This gives us an exact couple and so induces a bockstein spectral sequence. In particular, we get the following,

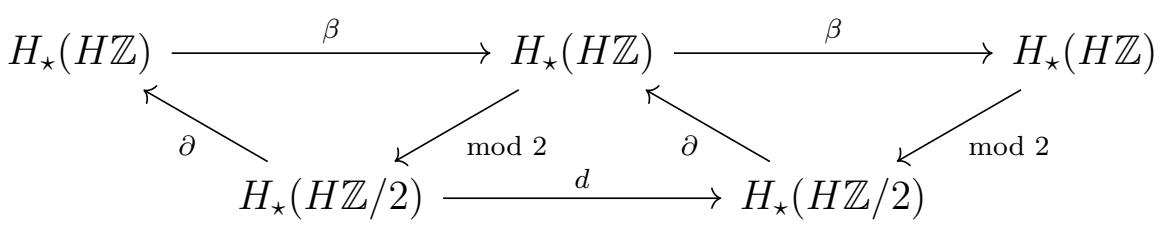

Notice that $2=0$ in $H_{\star}(H \mathbb{Z})$, and so we have that

$$
H_{\star}(H \mathbb{Z} / 2) \stackrel{\bmod 2}{\longrightarrow} H_{\star}(H \mathbb{Z} / 2)
$$

is injective, and so we have a short exact sequence

$$
0 \rightarrow H_{\star}(H \mathbb{Z}) \stackrel{\bmod 2}{\longrightarrow} H_{\star}(H \mathbb{Z} / 2) \stackrel{d}{\rightarrow} H_{\star}(H \mathbb{Z} / 2) \rightarrow 0 .
$$

Here $d$ is the dual of the steenrod operation $\mathrm{Sq}^{1}$. Notice that $H_{\star}(H \mathbb{Z})=\operatorname{ker}(d)$.

Lemma 3.11. The motivic cohomology of $H_{\star}(H \mathbb{Z})$ over $\mathrm{k}=\mathbb{C}$ is isomorphic to

$$
\mathbb{Z} / 2\left[\theta, \tau_{1}, \tau_{2}, \ldots, \xi_{1}, \xi_{2}, \ldots\right] /\left(\tau_{i}^{2}-\theta \xi_{i+1}\right)
$$

Proof. First one observes that $d\left(\tau_{0}\right)=1$ and $d\left(\tau_{i}\right)=\xi_{i}$ for $i \in \mathbb{Z}^{>0}$. Next, one 
observes that since $d$ commutes with the tate twist $\theta$, and since $\tau_{i}^{2}=\theta \xi_{i+1}$, we have

$$
0=2 \tau_{i} d\left(\tau_{i}\right)=d\left(\tau_{i}^{2}\right)=\theta d\left(\xi_{i+1}\right)
$$

Therefore $d\left(\xi_{i+1}\right)=0$. Now, as a $\mathbb{Z} / 2[\theta]$-algebra, the classes $\left\{\xi_{i}\right\}_{i=1}^{\infty}$, and the classes $\left\{c\left(\xi_{i}\right)\right\}_{i=1}^{\infty}$ both generate the same algebra. Looking now at the inductive formula for the conjugate of $\tau_{i}$, and aknowledging that $2=0$ in the coefficients, we have

$$
c\left(\tau_{k}\right)=\tau_{k}+c\left(\tau_{0}\right) \xi_{k}+c\left(\tau_{1}\right) \xi_{k-1}^{2}+\ldots+c\left(\tau_{k-1}\right) \xi_{1}^{2^{k-1}}
$$

First we notice that $c\left(\tau_{0}\right)=\tau_{0}$, and so $d\left(c\left(\tau_{0}\right)\right)=1$. I claim that $d\left(c\left(\tau_{i}\right)\right)=0$ for $i \in \mathbb{Z}^{>0}$. For $\tau_{1}$, we have that $c\left(\tau_{1}\right)=\tau_{1}+\tau_{0} \xi_{1}$. Taking the differential of each side, we have that

$$
d\left(c\left(\tau_{1}\right)\right)=d\left(\tau_{1}\right)+\tau_{0} d\left(\xi_{1}\right)+\xi_{1} d\left(\tau_{0}\right)=d\left(\tau_{1}\right)+\xi_{1}=\xi_{1}+\xi_{1}=0 .
$$

Now, by induction we can assume $d\left(c\left(\tau_{n-1}\right)\right)=0$. Therefore,

$$
\begin{array}{r}
d\left(c\left(\tau_{n}\right)\right)=d\left(\tau_{n}\right)+d\left(c\left(\tau_{0}\right) \xi_{n}\right)+d\left(c\left(\tau_{1}\right) \xi_{n-1}^{2}\right)+\ldots+d\left(c\left(\tau_{n-1}\right) \xi_{1}^{2^{n-1}}\right)= \\
d\left(\tau_{n}\right)+d\left(c\left(\tau_{0}\right) \xi_{n}\right)=d\left(\tau_{n}\right)+\xi_{n}=\xi_{n}+\xi_{n}=0 .
\end{array}
$$

Thus, $\operatorname{ker}(d)=\mathbb{Z} / 2\left[\theta, c\left(\tau_{1}\right), c\left(\tau_{2}\right), \ldots, c\left(\xi_{1}\right), c\left(\xi_{2}\right), \ldots\right]$. One can show that $c\left(\tau_{i}\right)^{2}=$ $\theta c\left(\xi_{i+1}\right)$. This proves the claim.

\subsubsection{The $\mathrm{Sq}^{1}$ cohomology}

Notice that the motivic steenrod operation $\mathrm{Sq}^{1}$ has the property that $\mathrm{Sq}^{1} \circ \mathrm{Sq}^{1}=0$. Therefore, we can think of $\mathrm{Sq}^{1}$ as a differential of $H^{\star}(\mathrm{MSLO})$. I will use the notation 
$H^{\star}\left(M ; \mathrm{Sq}^{1}\right)$ to denote the cohomology of the $\mathcal{A}^{\star}$ module $M$ with respect to the differential $M$.

Let $I=\left(\epsilon_{0}, s_{1}, \epsilon_{1}, s_{2}, \ldots, s_{k}, \epsilon_{k}\right)$ be a sequence where $\epsilon_{i} \in\{0,1\}$ and $s_{i}$ are nonnegative integers. Denote by $P^{I}$ the product

$$
P^{I}=\beta^{\epsilon_{0}} P^{s_{1}} \ldots P^{s_{k}} \beta^{\epsilon_{k}}
$$

A sequence $I$ is called admissible if $s_{i} \geq 2 s_{i+1}+\epsilon_{i}$. Monomials $P^{I}$ corresponding to admissible sequences are called admissible monomials. Here $\beta=\mathrm{Sq}^{1}$.

Lemma 3.12. Admissible monomials generate $\mathcal{A}^{\star}$ as a left $H^{\star}$-module.

Proof. See [Voe03].

Lemma 3.13. Suppose $I=\left(0, s_{1}, \ldots, s_{k}, 0\right)$ and $J=\left(0, t_{1}, \ldots, t_{r}, 0\right)$ with $s_{1}, s_{k}, t_{1}, t_{r} \in$ $\mathbb{Z}^{>0}$. Then $\beta P^{I} \neq P^{J} \beta$. Also, $\beta P^{s} \neq P^{t} \beta$ for $s, t \in \mathbb{Z}^{>0}$.

Proof. This follows immediately from Lemma lemma 3.12.

Lemma 3.14. $H^{\star}\left(\mathcal{A}^{\star} ; S q^{1}\right)=0$ and $H^{\star}\left(\mathcal{A}^{\star} / \mathcal{A}^{\star} S q^{1} ; S q^{1}\right)=H^{\star}$.

Proof. To prove the first statement, we notice $\operatorname{im}\left(\mathrm{Sq}^{1}\right)=\mathrm{Sq}^{1} \mathcal{A}^{\star}=\operatorname{ker}\left(\mathrm{Sq}^{1}\right)=$ $\mathrm{Sq}^{1} \mathcal{A}^{\star}$. For the second statement, we notice that $\operatorname{im}\left(\mathrm{Sq}^{1}\right)=\mathrm{Sq}^{1} \mathcal{A}^{\star} / \mathcal{A}^{\star} \mathrm{Sq}^{1}$. Since $\mathrm{Sq}^{1} \mathcal{A}^{\star} / \mathcal{A}^{\star} \mathrm{Sq}^{1}$ is clearly in both the kernel and image of $\mathrm{Sq}^{1}$, and using Lemma lemma 3.13, we know that if $I=\left(0, s_{1}, \ldots, s_{k}, 0\right)$ with $s_{1}, s_{k} \in \mathbb{Z}^{>0}$ or $I=(s), s \in \mathbb{Z}^{>0}$, then $\mathrm{Sq}^{1} P^{I} \notin \mathcal{A}^{\star} \mathrm{Sq}^{1}$. We have shown what happens to admissible monomials. We only have to look at what happens to elements of $H^{\star}$. Clearly these elements get sent to zero since they commute with the $\mathrm{Sq}^{1}$ operation. Since elements of $H^{\star}$ are clearly not in the image of $\mathrm{Sq}^{1}$, it follows that $H^{\star}\left(\mathcal{A}^{\star} / \mathcal{A}^{\star} \mathrm{Sq}^{1}\right)=H^{\star}$.

We will need the following result. But first, from [SV14] we have the following proposition. 
Proposition 3.15. Recall that $H^{\star}\left(B O_{n_{+}}\right) \cong H^{\star}\left[w_{1}, \ldots, w_{n}\right]$ as an $H^{\star}$-module. If -1 is a square in $\mathrm{k}$, then

$$
S q^{k}\left(w_{m}\right)=\sum_{j=0}^{k}\left(\begin{array}{c}
m-k \\
j
\end{array}\right) w_{k-j} w_{m+j} .
$$

The Cartan formula over $\mathrm{k}=\mathbb{C}$ gives the following.

Proposition 3.16. Let $\tau$ be the tate twist of degree $\alpha-1$ in $H^{\star}$, and let $H^{\star}\left(B O_{n_{+}}\right) \cong$ $H^{\star}\left[w_{1}, \ldots, w_{n}\right]$. We define

$$
\epsilon_{i, j}=\left\{\begin{array}{rr}
1 & k \text { is even and } i, j \text { are odd } \\
0 & \text { otherwise. }
\end{array}\right.
$$

If -1 is a square in $\mathrm{k}$, then

$$
S q^{k}\left(w_{r} w_{s}\right)=\sum_{i+j=k} \tau^{\epsilon_{i, j}} S q^{i}\left(w_{r}\right) S q^{j}\left(w_{s}\right)
$$

Proof. This follows from the formulas given in [Voe03], along with relations between the geometric and simplicial classifying spaces of $O_{n}$ found in [SV14].

Lemma 3.17. $S q^{1} t_{n}=0$ where $t_{n} \in H^{\star}\left(\operatorname{Thom}\left(B S O_{n}\right)\right)$ is the thom class.

Proof. Let $H^{\star}\left(B O_{n+}\right)=H^{\star}\left[w_{1}, \ldots, w_{n}\right]$. Recall that by proposition $3.3, H^{\star}\left(B S O_{n+}\right)$ can be identified with $H^{\star}\left[w_{2}, w_{3}, \ldots, w_{n}\right] \subset H^{\star}\left(B O_{n+}\right)$. Recall also that there is a thom isomorphism

$$
H^{\star}\left(B S O_{n+}\right) \smile w_{n} \cong H^{\star}\left(\operatorname{Thom}\left(B S O_{n_{+}}\right)\right.
$$

Therefore, $\mathrm{Sq}^{1}\left(t_{n}\right)$ can be identified with $\mathrm{Sq}^{1}\left(w_{n}\right)$ under eq. (3.1) and so we can work out the steenrod operation on $H^{\star}\left(\operatorname{Thom}\left(B S O_{n}\right)\right)$ by comparison with $H^{\star}\left(B O_{n+}\right)$. In particular, $\mathrm{Sq}^{1}\left(w_{n}\right)=w_{n} w_{1}$. Since $w_{1}=0$ in $H^{\star}\left(B S O_{n+}\right)$, the claim follows. 
Since $H^{\star}(\mathrm{MSLO})$ is an $\mathcal{A}^{\star}$ module, we can compute its $\mathrm{Sq}^{1}$ cohomology.

Proposition 3.18. $H^{\star}\left(H^{\star}(\mathrm{MSLO}) ; S q^{1}\right)=H^{\star}\left[u_{2}^{2}, u_{4}^{2}, u_{6}^{2}, \ldots\right]$.

Proof. By Lemma lemma 3.17, $\mathrm{Sq}^{1}$ commutes with the thom isomorphism. Therefore it is enough to show that $H^{\star}\left(H^{\star}(\mathrm{BSO}) ; \mathrm{Sq}^{1}\right)=H^{\star}\left[w_{2}^{2}, w_{4}^{2}, w_{6}^{2}, \ldots\right]$. We note that $\mathrm{Sq}^{1}\left(w_{2 n}\right)=w_{2 n+1}$. From this it follows that $H^{\star}\left[u_{3}, u_{5}, u_{7}, \ldots\right] \subset \operatorname{im}\left(\mathrm{Sq}^{1}\right)$. This implies that the only elements which can be in the kernel but not in the image of $\mathrm{Sq}^{1}$ are $H^{\star}\left[w_{2}^{2}, w_{4}^{2}, w_{6}^{2}, \ldots\right] \subset H^{\star}(\mathrm{BSO})$. Noting that $\mathrm{Sq}^{1}\left(w_{2 n}^{2}\right)=0$ for all $n$, the claim follows.

\subsubsection{A motivic version of Wall's Theorem}

Lemma 3.19. The morphism of $\mathcal{A}^{\star}$-modules,

$$
\mathcal{A}^{\star} \rightarrow H^{\star}(\mathrm{MSLO})
$$

given by $a \mapsto a \cdot 1$ where 1 denotes the thom class $t_{0} \in H^{0,0}$ (MSLO) has kernel $J=\mathcal{A}^{\star} S q^{1}$.

Proof. To simplify notation, we write $\mathcal{A}^{\star} / \beta:=\mathcal{A}^{\star} / \mathcal{A}^{\star} \mathrm{Sq}^{1}$.

First, it is clear that $\operatorname{Sq}^{i}\left(w_{j}\right)=0$ if $i>j$ by proposition 3.15. If $i \leq j$, then $\mathrm{Sq}^{1}\left(w_{j}\right)$ is a sum of monomials $w_{k} w_{l}$ with $k, l<2 j$. The monomials $\mathrm{Sq}^{i_{n}} \ldots \mathrm{Sq}^{i_{1}}$ with $i_{n} \geq 2 i_{n-1}$ and $i_{1}>1$ form an $H^{\star}$-module basis for $\mathcal{A}^{\star} / \beta$. Therefore, it is enough to show that the polynomials $\mathrm{Sq}^{i_{n}} \ldots \mathrm{Sq}^{i_{1}}(t)$ are linearly independent in $H^{\star}(\mathrm{MSLO})$. Let $I=\left(i_{k}, \ldots, i_{1}\right)$ with $i_{s} \geq 2 i_{s-1}$ and $i_{1}>1$. We will order the monomials $w^{I}=$ $w^{i_{k}} w^{i_{k-1}} \ldots w^{i_{1}}$ lexicographically. For example, $w_{8} w_{4}$ is of higher order than $w_{4} w_{2}$ and $w_{8} w_{2}$, but lower order than $w_{8} w_{4} w_{2}$ and $w_{10} w_{2}$. By induction, we will assume that $\mathrm{Sq}^{i_{n-1}} \ldots \mathrm{Sq}^{i_{1}}(t)=w_{i_{n-1}} \ldots w_{i_{1}} t+$ lower order terms.

Now suppose that $w_{j_{n-1}} \ldots w_{j_{1}} t \in H^{\star}(\mathrm{MSLO})$ is such that $j_{n-1} \geq j_{n-1} \geq \ldots \geq j_{1}$. If $i \geq 2 j_{n-1}$, then we will show $\operatorname{Sq}^{i}\left(w_{j_{n-1}} \ldots w_{j_{1}} t\right)=w_{i} w_{j_{n-1}} \ldots w_{j_{1}} t+$ lower order terms. 
Using the Cartan formula, we have

$$
\begin{array}{r}
\operatorname{Sq}^{i}\left(w_{j_{n-1}} \ldots w_{j_{1}} t\right)=\mathrm{Sq}^{i}(t) \cdot w_{j_{n-1}} \ldots w_{j_{1}}+\text { lower order terms } \\
=w_{i} w_{j_{n-1}} \ldots w_{j_{1}} t+\text { lower order terms. }
\end{array}
$$

This proves the theorem.

Theorem 3.20. $H^{\star}(\mathrm{MSLO})$ is a wedge sum of suspensions of $\mathcal{A}^{\star}$ and $\mathcal{A}^{\star} / \mathcal{A}^{\star} S q^{1}$.

Proof. To simplify notation, we write $M:=H^{\star}(\mathrm{MSLO})$, and $\mathcal{A}^{\star} / \beta:=\mathcal{A}^{\star} / \mathcal{A}^{\star} \mathrm{Sq}^{1}$. Notice that $\mathrm{Sq}^{1}$ acts on any $\mathcal{A}^{\star}$-module as a differential. This is immediate from the fact that $\mathrm{Sq}^{1} \mathrm{Sq}^{1}=0$. Therefore, for any $\mathcal{A}^{\star}$-module $P$ we can define the $\mathrm{Sq}^{1}$ cohomology $H^{\star}\left(P ; \mathrm{Sq}^{1}\right)$ of $P$. We will be working with $H^{\star}$-modules, and so this cohomology theory will have coefficients in $H^{\star}$. As we have already shown in Lemma lemma 3.14, $H^{\star}\left(\mathcal{A}^{\star} ; \mathrm{Sq}^{1}\right)=0$, and $H^{\star}\left(\mathcal{A}^{\star} / \beta ; \mathrm{Sq}^{1}\right)=H^{\star}$.

We will now use this cohomology theory to define a map from a wedge sum of suspensions of $\mathcal{A}^{\star} / \beta$ to $M$ which will induce an isomorphism in $\mathrm{Sq}^{1}$ cohomology.

Choose classes $\left\{x_{\alpha}\right\}_{\alpha \in I} \in M$ whose images in $H^{\star}\left(M ; \mathrm{Sq}^{1}\right)$ form a basis. By Proposition proposition 3.18 , we can choose the classes $u_{2}^{2}, u_{4}^{2}, \ldots \in H^{\star}(\mathrm{MSLO}) \cong$ $H^{\star}\left[u_{2}, u_{3}, u_{4}, \ldots\right]$. The $x_{\alpha}$ are killed by $\mathrm{Sq}^{1}$ and so we can define a map

$$
\phi_{1}: \bigoplus_{\alpha \in I} \mathcal{A}^{\star} / \beta\left[-\operatorname{deg}\left(x_{\alpha}\right)\right] \rightarrow M
$$

Next, we define

$$
\overline{\mathcal{A}^{\star}}:=\left\{\text { admissible monomials } x \in \mathcal{A}^{\star}|| x \mid>0\right\} \text {. }
$$


Using this definition, we define

$$
\bar{M}:=M / \overline{\mathcal{A}^{\star}} M .
$$

Notice that $\bigoplus_{\alpha \in I} \mathcal{A}^{\star} / \beta\left[-\operatorname{deg}\left(x_{\alpha}\right)\right] \cong \mathcal{A}^{\star} / \beta \otimes_{H^{\star}} C$ for $C=H^{\star}\left[u_{2}^{2}, u_{4}^{2}, \ldots\right]$. We consider the projection map

$$
M \stackrel{\pi}{\rightarrow} \bar{M}
$$

We then choose an $H^{\star}$-submodule $Z \subset M$ such that $\pi_{\left.\right|_{Z}}$ is injective, and

$$
\bar{M} \cong \pi\left(\phi_{1}\left(\mathcal{A}^{\star} / \beta \otimes C\right)\right) \oplus \pi(Z)
$$

Set

$$
N=\mathcal{A}^{\star} / \beta \otimes C \oplus \mathcal{A}^{\star} \otimes Z
$$

The natural map

$$
\phi_{2}: \mathcal{A}^{\star} \otimes Z \rightarrow M
$$

gives a map

$$
\Phi:=\phi_{1} \oplus \phi_{2}: N \rightarrow M
$$

Writing $N=\mathcal{A}^{\star} / \beta \otimes C \oplus \mathcal{A}^{\star} \otimes Z$, we let $N_{i}$ denote the $\mathcal{A}^{\star}$-submodule of $N$ given by $N=\mathcal{A}^{\star} / \beta \otimes C_{i} \oplus \mathcal{A}^{\star} \otimes Z_{i}$. Here $C_{i}$ and $Z_{i}$ denotes all elements in $C$ and $Z$ respectively of total degree $i$. We say the class $x$ with degree $n+m \alpha$ has total degree $n+m$. We define $M_{i}$ to be the image of $N_{i}$ under the map $\Phi$. We then define $N^{(n)}$ and $M^{(n)}$ to be $\oplus_{i \leq n} N_{i}$ and $\oplus_{i \leq n} \Phi\left(N_{i}\right)$ respectively.

We will show by induction that the map $\Phi: N^{(n)} \rightarrow M^{(n)}$ is an isomorphism. Starting with $n=0, N^{(0)}=\mathcal{A}^{\star} / \beta$ and $M^{(0)}=\mathcal{A}^{\star} \cdot t$, where $t$ is the thom class. By Lemma lemma 3.19 this map is an isomorphism. Suppose we have proved $\Phi$ : $N^{(n-1)} \rightarrow M^{(n-1)}$ is an isomorphism and let $\lambda: N / N^{(n-1)} \rightarrow M / M^{(n-1)}$ be the map 
induced by $\Phi$. We will show $\lambda_{\left.\left.\right|_{(N(n) / N}(n-1)\right)}$ is injective. Let $P \subset N$ be the subspace generated by elements of the form $c, z, \mathrm{Sq}^{1}(z)$ for $c \in C_{n}, z \in Z_{n}$. We can regard $P$ as an $H^{\star}$-submodule of $N / N^{(n-1)}$.

We will first prove that $\lambda_{\left.\right|_{P}}$ is injective. Notice that since $H^{\star}\left(\mathcal{A}^{\star} ; \mathrm{Sq}^{1}\right)=0$, the map

$$
\Phi^{*}: H^{\star}\left(N ; \mathrm{Sq}^{1}\right) \rightarrow H^{\star}\left(M ; \mathrm{Sq}^{1}\right)
$$

is still an isomorphism. Since

$$
\Phi: N^{(n-1)} \rightarrow M^{(n-1)}
$$

is an isomorphism by induction, it follows that

$$
\lambda^{*}: H^{\star}\left(N / N^{(n-1)} ; \mathrm{Sq}^{1}\right) \rightarrow H^{\star}\left(M / M^{(n-1)} ; \mathrm{Sq}^{1}\right)
$$

is also an isomorphism.

Suppose $v \in P$ and $\lambda(v)=0$. Notice that the total dimension of $v$ is $n$ or $n+1$. We will consider the two cases separately. If the total dimension of $v$ is $n$; then $v=c+z$ for $c \in C_{n}, z \in Z_{n} . \lambda(v)=0$ implies $\Phi(c+z) \in M_{n}^{(n-1)}$. By choice of $Z$, $z=0$. Then, $v=c$, and so $\lambda(c)=0$. Since $\lambda^{*}$ is an isomorphism, it follows that $\mathrm{Sq}^{1}(c)=0$, and $c=\mathrm{Sq}^{1}\left(c^{\prime}\right)$ for some $c^{\prime} \in\left(N / N^{(n-1)}\right)_{n-1}$. But $\left(N / N^{(n-1)}\right)_{n-1}=0$, and so $c^{\prime}=0$, which implies $c=0$.

Now, suppose that the total dimension of $v$ is $n+1$ : then $v=\operatorname{Sq}^{1}(z)$, some $z \in Z_{n}$. If $\lambda(v)=0$, then $\operatorname{Sq}^{1}(\lambda(z))=0$. But, this means $\lambda(z)=\lambda(c)+\operatorname{Sq}^{1}\left(z^{\prime}\right)$ for some $z^{\prime} \in\left(M / M^{(n-1)}\right)_{n-1}$. But, this means $z^{\prime}=0$. Therefore we reduce to the previous case.

Now, returning to the induction step, we have that the multiplication map

$$
\mu: \mathrm{MSLO} \wedge \mathrm{MSLO} \rightarrow \mathrm{MSLO}
$$


induces a coproduct map

$$
\mu^{*}: H^{\star}(\mathrm{MSLO}) \rightarrow H^{\star}(\mathrm{MSLO}) \otimes_{H^{\star}} H^{\star}(\mathrm{MSLO})
$$

We define a projection map

$$
p: M \rightarrow M / M^{(n-1)}
$$

Let $u \in C_{n} \oplus Z_{n}$. Then $\mu^{*} \Phi(u)=1 \otimes_{H^{\star}} \Phi(u)$ modulo $M \otimes_{H^{\star}} M^{(n-1)}$. Therefore, for any $v \in P$ we have

$$
\left(1 \otimes_{H^{\star}} p\right) \mu^{*} \Phi(v)=1 \otimes_{H^{\star}} \lambda(v)
$$

Now choose a basis $c_{1}, c_{2}, \ldots, c_{r}$ for $C_{n}, z_{1}, z_{2}, \ldots, z_{s}$ for $Z_{n}$. Then we can give $P$ a basis $\left\{v_{i}\right\}=\left\{c_{1}, \ldots, c_{r}, z_{1}, z_{2}, \ldots, z_{s}, \mathrm{Sq}^{1}\left(z_{1}\right), \mathrm{Sq}^{1}\left(z_{2}\right), \ldots, \mathrm{Sq}^{1}\left(z_{s}\right)\right\}$. Any $v \in N^{(n)} / N^{(n-1)}$ then has a unique expression in the form $v=\sum_{i} a_{i} v_{i}$ for $a_{i} \in \mathcal{A}^{\star} \backslash \mathcal{A}^{\star} \mathrm{Sq}^{1} \cup\{0\}$. Now, we let $m$ denote the maximum total dimension of all of the $a_{i}$. Next, we let $\left\{a_{i_{1}}, a_{i_{2}}, \ldots, a_{i_{v}}\right\}$ denote all of the $a_{j}$ of total dimension $m$.

Notice that if $\lambda(v)=0$, then $\Phi(v) \in M^{(n-1)}$ and hence

$$
0=\left(1 \otimes_{H^{\star}} p\right) \mu^{*} \Phi(v)=\sum a_{i_{j}} \cdot t \otimes_{H^{\star}} \lambda\left(v_{i_{j}}\right)+\sum b_{k} \cdot t \otimes_{H^{\star}} m_{k},
$$

some $m_{k} \in M, b_{k} \in \mathcal{A}^{\star}$ with $\operatorname{dim} b_{k}<m$. However, we showed that $\lambda_{\left.\right|_{P}}$ is injective, and so the $\lambda\left(v_{i_{j}}\right)$ are linearly independent. and hence $a_{i_{j}} \cdot t=0$ for all $j$. But, $a_{i_{j}} \cdot t=0$ implies $a_{i_{j}} \in \mathcal{A}^{\star} \mathrm{Sq}^{1}$. This is a contradiction, and so $\lambda(v)=0$ implies $v=0$.

Corollary 3.21. Over the field $\mathrm{k}=\mathbb{C}$,

$$
H_{\star}(\mathrm{MSLO}) \cong H_{\star}(H \mathbb{Z} / 2) \otimes_{\mathbb{Z} / 2[\theta]} C \oplus \mathcal{A}_{\star} \otimes_{\mathbb{Z} / 2[\theta]} Z
$$


Here $C$ is the algebra $\mathbb{Z} / 2\left[\theta, x_{4}, x_{8}, \ldots\right]$ where the $x_{4 i}$ are generators of degree $2(1+\alpha) i$. $Z$ is a $\mathbb{Z} / 2[\theta]$ polynomial algebra.

\subsubsection{The homotopy type of MSLO}

Once we know that the motivic $\mathbb{Z} / 2$ homology of MSLO is a wedge sum of suspensions of $\mathcal{A}^{\star}$ and $\mathcal{A}^{\star} / \mathcal{A}^{\star} \mathrm{Sq}^{1}$, we can again construct a map

$$
\mathrm{MSLO} \rightarrow \bigvee_{i \in I} H \mathbb{Z} / 2\left[r_{i}\right] \bigoplus \bigvee_{j \in J} H \mathbb{Z}\left[s_{j}\right]
$$

which is an equivalence on motivic $\mathbb{Z} / 2$ homology. Then, by applying the Nakayama lemma and the motivic Hurewicz theorem [Bac15] to show that the map is a homotopy equivalence.

\subsubsection{The dimension of the $H \mathbb{Z} / 2$ suspensions}

Let $\mathrm{N}$ be an $H_{\star}$-module with basis $\mathfrak{N}$. Assume $\mathfrak{N}=\bigcup_{n, m \geq 0} \mathfrak{N}_{n+m \alpha}$, where $\mathfrak{N}_{n+m \alpha}$ is a finite set consisting of all basis elements in degree $n+m \alpha$. Call the motivic spectrum E special if it satisfies these assumptions.

If $\mathrm{E}$ is special, then we can define a polynomial $f \in \mathbb{Z}[[x, y]]$ by considering the formal sum $\sum_{n+m=j} r_{n+m \alpha}$. We then map this to a polynomial in $f \in \mathbb{Z}[[x, y]]$ by sending $r_{n+m \alpha} \mapsto x^{n} y^{m}$. In this way, for $\mathrm{E}$ special, we can define $|\mathrm{N}|:=f$.

In considering MSLO, it is clear where the $H \mathbb{Z}$ suspensions must live; they consist of suspensions of degrees corresponding to all monomials in the variables $x_{1}, x_{2}, \ldots$ where $x_{i}$ has degree $2 i(1+\alpha)$. The tricky part is to see where all the $H \mathbb{Z}$ suspensions must live.

The way we will figure this out is through a combinatorial counting argument. To explain the idea behind the counting argument, I will first give a simple example.

Example 3.22. Suppose we wanted to know where the $H \mathbb{Z} / 2$ suspensions are in the 
dual motivic steenrod algebra $\mathcal{A}_{\star}$. Notice that as an $H^{\star}$-module,

$$
\mathcal{A}_{\star} \cong H^{\star}\left[\tau_{0}, \tau_{1}, \ldots, \xi_{1}, \xi_{2}, \ldots\right] /\left(\tau_{i}^{2} \mid 0 \leq i\right)
$$

In other words, we can sort of pretend that $\tau_{i}^{2}=0$ in $\mathcal{A}_{\star}$ since we only care about the $H \mathbb{Z} / 2$ module structure. We also only care about how many suspensions we have in each degree. Letting the monomial $x^{n} y^{m}$ represent suspension by $n+m \alpha$, we can consider the formal sum of all monomials in $\mathcal{A}_{\star}$ of the form $\tau_{i_{1}}^{\epsilon_{1}} \tau_{i_{2}}^{\epsilon_{2}} \ldots \tau_{i_{n}}^{\epsilon_{n}} \xi_{j_{1}}^{r_{1}} \xi_{j_{2}}^{r_{2}} \ldots \xi_{j_{m}}^{r_{m}}$ where $\epsilon_{i} \in\{0,1\}$ and $r_{i} \in \mathbb{Z}^{\geq 0}$ modulo their degree. For example, we represent $\tau_{1} \xi_{1}$ by $x^{2} y^{1} \cdot x y=x^{3} y^{2}$. Therefore, we obtain the formal sum

$$
\left|\mathcal{A}_{\star}\right|=\frac{\prod_{i \geq 0}\left(1-x^{2^{i+1}} y^{2^{i+1}-2}\right)}{\prod_{i \geq 0}\left(1-x^{2^{i+1}-1} y^{2^{i+1}-1}\right) \prod_{i \geq 0}\left(1-x^{2^{i}} y^{2^{i}-1}\right)} .
$$

Here formal sum refers to the taylor series expansion about the origin $(0,0)$. Using a computer software program such as matlab, or by expanding by hand, one can deduce where the suspensions of $H \mathbb{Z} / 2$ live. Similarly, we can deduce,

$$
\left|\mathcal{A}_{\star} / \mathcal{A}_{\star} S q^{1}\right|=\frac{\prod_{i>0}\left(1-x^{2^{i+1}} y^{2^{i+1}-2}\right)}{\prod_{i \geq 0}\left(1-x^{2^{i+1}-1} y^{2^{i+1}-1}\right) \prod_{i>0}\left(1-x^{2^{i}} y^{2^{i}-1}\right)} .
$$

We end up with the following formula.

Proposition 3.23. Consider the taylor series expansion around the point $(x, y)=$ $(0,0)$ of the following rational binomial polynomial:

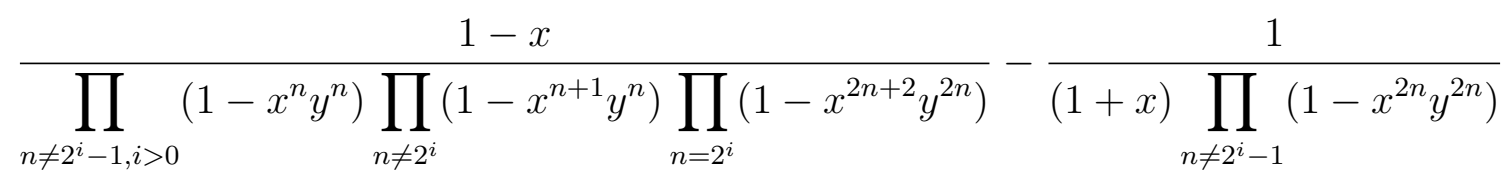


Its coefficients tells us how many suspensions of $H \mathbb{Z} / 2$ we have in a given degree. For example, the term $c x^{n} y^{m}$ means that we have $c$ suspensions of $H \mathbb{Z} / 2$ in degree $n+m \alpha$.

Proof. First we notice that

$$
\left|H_{\star}(\mathrm{MSLO})\right|=\frac{1}{\prod_{i \geq 0}\left(1-x^{i} y^{i}\right) \prod_{i>0}\left(1-x^{i+1} y^{i}\right)} .
$$

We also have that the degree suspensions of the $H \mathbb{Z}$ is equal to $\left|H_{\star}\left[x_{1}, x_{2}, ..\right]\right|$ where $\left|x_{i}\right|=2 i(1+\alpha)$. It follows that

$$
\left|\mathrm{MSLO}_{\star}\right|=\frac{1}{\left|\mathcal{A}_{\star}\right|}\left(\left|H_{\star}(\mathrm{MSLO})\right|-\left|H_{\star}\left[x_{1}, x_{2}, \ldots\right]\right|\right) .
$$

The claim follows. 


\section{CHAPTER IV}

\section{A motivic analogue of MR}

\subsection{MGLR, an analogue of MR}

There is a $C_{2}$-equivariant spectrum belonging to classical topology which was constructed by Landweber. The coefficients of this spectrum were computed by P. $\mathrm{Hu}$ and I. Kriz in [HK01]. The coefficients of this spectrum are bigraded. While the bigrading given in [HK01] is $\mathrm{MR}_{*+*^{\prime} \alpha}$, we will use $\sigma$ grading instead of $\alpha$. The reason for this is that the authors of [HK01] used the $\alpha$ to signify the relationship between motivic homotopy theory and classical $C_{2}$-equivariant homotopy theory. The topological realization functor over $\mathbb{R}$ sends motivic $\alpha$ grading to the $C_{2}$ grading. However, in the present case, we want to stress the relationship between $C_{2}$ motivic homotopy theory and $C_{2}$ classical homotopy theory using the topological realization over $\mathbb{C}$.

In this chapter we discuss a $C_{2}$-equivariant motivic spectrum MGLR which was constructed by P. Hu and I. Kriz in [HKO11]. There is a complex topological realization functor $t_{C_{2}}^{\mathbb{C}}$ for $C_{2}$-equivariant motivic spectra, and $t_{C_{2}}^{\mathbb{C}}(\mathrm{MGLR})=\mathrm{MR}$.

One should think of MGLR as a motivic analogue of MR. Roughly speaking, the spectrum MR can be thought of as complex cobordism MU endowed with a $C_{2}$ action. At its heart, $\mathrm{MU}$ is built from the classifying spaces $B U_{n}$, where $U_{n}$ denotes the $n$ dimensional unitary group. We get an involution on this group given by $A \leftrightarrow \bar{A}^{T}$. 
The groups $U_{n}$ equipped with this involution action determine the construction of MR. If one wanted to mimic this construction motivically, he would immediately be faced with a problem; complex conjugation is not algebraic. A priori this means that the groups $U_{n}$ are not definable; however, it turns out that over the complex numbers, $U_{n} \cong G L_{n}(\mathbb{C})$. In fact, the motivic analogue of $\mathrm{MU}$ is the well known algebraic cobordism MGL.

In analogy with MR, MGLR should be thought of as algebraic cobordism MGL endowed with a $C_{2}$ action. Consider the symmetric bilinear form

$$
b\left(\left(x_{1}, \ldots, x_{2 n}\right),\left(y_{1}, \ldots, y_{2 n}\right)\right)=\sum_{i=1}^{n} x_{2 i} y_{2 i-1}+x_{2 i-1} y_{2 i} .
$$

Then for any $A \in G L_{2 n}(\mathrm{k})$, there exists a unique matrix $A^{T_{b}}$ for which $b(A x, y)=$ $b\left(x, A^{T_{b}} y\right)$ for all $x, y \in \mathrm{k}^{2 n}$. The $C_{2}$ action of MGLR is induced from the involution action $A \leftrightarrow\left(A^{T_{b}}\right)^{-1}$.

\subsubsection{The $\lambda$ twist}

In [HK01], the authors show that MR completed at 2 splits as a wedge sum of suspensions of a spectrum BPR whose suspensions are in degrees $m_{i}(1+\sigma)$ for $m_{i} \neq 2^{i+1}-1, \Phi^{C_{2}}(\mathrm{BPR})=H \mathbb{Z} / 2$, and nonequivariantly BPR $=\mathrm{BP}$. This splitting comes from applying the Quillen idempotent to the formal group law on $\mathrm{MR}_{*(1+\sigma)}$. From this, it follows that $\mathrm{MR}_{\star}$ is freely generated by generators $x_{n}$ of degree $n(1+\sigma)$ for $n \neq 2^{i+1}-1$ as a $\mathrm{BPR}_{\star}$ algebra. One could ask whether MGLR splits as a wedge sum of suspensions of BPGLR, with $\Phi^{C_{2}}(\mathrm{BPGLR})=H \mathbb{Z} / 2$ and BPGLR $=$ BPGL nonequivariantly, in such a way that $M G L R_{\star}$ is free as a BPGLR $R_{\star}$ algebra. Unfortunately, there does not appear to be any way to construct such a splitting. However, there exists an element $\lambda \in \pi_{1-\sigma+\sigma \alpha-\alpha}(\mathrm{MGLR})$. If we invert this element, then we get a formal group law and we can use the Quillen idempotent construction 
to get a splitting. First, let us elaborate on this mysterious element $\lambda$.

In the topological setting there is the notion of real-oriented spectra and it turns out that MR is universal among real-oriented spectra. There is also a notion of realorientation found in [HKO11]. Following notation in [HKO11], we define $\widetilde{X}$ to be the functorial fibrant replacement of $\bar{X}$, the reduced suspension of $X$.

Definition 4.1. A $C_{2}$-equivariant ring spectrum $E$ is real-oriented if the following two conditions are satisfied. Here MGLR(1) will denote the first term of the prespectrum defining MGLR.

1. The unit in $E^{\star}\left(S^{1+\sigma \alpha+\sigma+\alpha}\right)$ restricts to the unit $\phi_{E}$ of $E^{\star}(\operatorname{MGLR}(1))$.

2. The map $S^{2+2 \sigma \alpha} \simeq \widetilde{\mathbb{G}_{m}^{1 / z}} \wedge \widetilde{\mathbb{G}_{m}^{1 / z}} \rightarrow \widetilde{\mathbb{G}_{m}^{1 / z}} \times \widetilde{\mathbb{G}_{m}^{1 / z}} \rightarrow B\left(\mathbb{G}_{m}^{1 / z} \times \mathbb{G}_{m}^{1 / z}\right) \rightarrow B G L_{2} \rightarrow$ $\operatorname{MGLR}(1)$ with representative $\omega \in \pi_{2+2 \sigma \alpha}$ composes with $\phi_{E}$ to give a unit $\lambda_{E}$.

Whenever this is satisfied we get many results analogous to those found in [HK01].

Theorem 4.2. If the $C_{2}$-equivariant ring spectrum $E$ is real-oriented, then $E^{\star}\left(B \mathbb{G}_{m}^{1 / z}\right)=E^{\star}[u]$ where $\operatorname{deg}(u)=-(1+\sigma \alpha)$.

Unfortunately, it is not clear whether or not MGLR satisfies definition 4.1. Clearly MGLR satisifies condition 1 of definition 4.1. However, it is not clear that $\lambda_{\mathrm{MGLR}}$ is invertible. Using the methods of [EKMM07] we can "invert" $\lambda_{M G L R}$ to construct a spectrum $\lambda^{-1}$ MGLR satisfying the conditions of definition 4.1. The formal group law of theorem 4.2 then gives a canonical map

$$
\mathrm{L} \rightarrow \lambda^{-1} \mathrm{MGLR}_{*(1+\sigma \alpha)}
$$

Here $\mathrm{L}$ denotes the Lazard ring.

Notice that the topological realization functor over $\mathbb{C}$, which we denote by $t^{\mathbb{C}}$, is a symmetric monoidal functor, and so applied to the spectrum MGLR, we get a ring 
homomorphism

$$
\mathrm{MGLR}_{\star} \rightarrow \mathrm{MR}_{\star}
$$

One can show that $\lambda_{\mathrm{MGLR}}$ is sent to the unit 1 under this ring homomorphism, and so we get a ring homomorphism

$$
\lambda^{-1} \mathrm{MGLR}_{\star} \rightarrow \mathrm{MR}_{\star}
$$

Since the homomorphism $t^{\mathbb{C}}$ sends $1+\sigma \alpha$ grading to $1+\sigma$ grading, and since $\lambda^{-1} \mathrm{MGLR}_{*(1+\sigma \alpha)} \subset \lambda^{-1} \mathrm{MGLR}_{\star}$ and $\mathrm{MR}_{*(1+\sigma)} \subset \mathrm{MR}_{\star}$ are commutative rings, we have the following result.

Lemma 4.3. The restriction of the ring homomorphism eq. (4.1) to $\lambda^{-1} \mathrm{MGLR}_{*(1+\sigma \alpha)}$ induced by the topological realization functor $t^{\mathbb{C}}$ sends the formal group law on $\lambda^{-1} \mathrm{MGLR}_{\star}$ to the formal group law on $\mathrm{MR}_{\star}$.

Proof. This is clear since $t^{\mathbb{C}}\left(B \mathbb{G}_{m}^{1 / z}\right)=B S^{\sigma}$.

Since MGLR is an $\mathrm{E}_{\infty}$-ring spectrum, we may apply constructions as in [EKMM07]. In particular, we may "kill" or "invert" the image of any sequence of elements of $L$ in the spectrum $\lambda^{-1} \mathrm{MGLR}$. The ring $\mathrm{MGL}_{*(1+\alpha)}=\mathrm{MU}_{2 *}$ is the universal formal group law and so the generator $x_{i}$ of degree $i(1+\alpha)$ is sent to an element of degree $i(1+\sigma \alpha)$.

Theorem 4.4. $\Phi^{C_{2}}\left(\lambda^{-1} \mathrm{MGLR}\right)=\theta^{-1} \mathrm{MGLO}$.

Proof. Recall that $\lambda$ is the map

$$
\begin{aligned}
S^{2+2 \sigma \alpha} \simeq \widetilde{\mathbb{G}_{m}^{1 / z}} \wedge \widetilde{\mathbb{G}_{m}^{1 / z}} \rightarrow \widetilde{\mathbb{G}_{m}^{1 / z}} \times \widetilde{\mathbb{G}_{m}^{1 / z}} \rightarrow B\left(\mathbb{G}_{m}^{1 / z} \times \mathbb{G}_{m}^{1 / z}\right) & \rightarrow B G L_{2} \rightarrow \operatorname{MGLR}(1) \\
& \rightarrow \Sigma^{1+\sigma+\sigma \alpha+\alpha} \text { MGLR }
\end{aligned}
$$


After taking geometric fixed points, this becomes a map,

$$
S^{2} \simeq S^{1} \wedge S^{1} \rightarrow S^{1} \times S^{1} \rightarrow B(\mathbb{Z} / 2 \times \mathbb{Z} / 2) \rightarrow B O_{2} \rightarrow \mathrm{MGLO}(1) \rightarrow \Sigma^{1+\alpha} \mathrm{MGLO}
$$

This map is nonzero, and it realizes as an element of degree $1-\alpha$ in $\pi_{\star}(\mathrm{MGLO})$. Notice that there exists exactly one element in $\pi_{\star}(\mathrm{MGLO})$ of degree $1-\alpha$, the tate twist. Therefore, the coefficients of $\Phi^{C_{2}}\left(\lambda^{-1} \mathrm{MGLR}\right)$ is $\pi_{\star}\left(\theta^{-1} \mathrm{MGLO}\right) \cong \pi_{*}(\mathrm{MO})\left[\theta^{ \pm 1}\right]$.

\section{Corollary 4.5. MGLR $\nsucceq \lambda^{-1}$ MGLR.}

Proof. Since MGLR and $\lambda^{-1}$ MGLR are not equal on geometric fixed points, they cannot possibly be equal equivariantly.

It is interesting to note that while inverting $\lambda$ has the effect of inverting the tate twist $\theta$ under the geometric fixed points map, it is not the case that $\theta$ is inverted under the forgetful map MGLR $\rightarrow$ MGL which thinks of the structure nonequivariantly. The reason for this is the forgetful map sends $\sigma$ and $\sigma \alpha$ grading to 1 and $\alpha$ respectively. Therefore, $\lambda$ gets sent to the unit under this map. In more detail,

Theorem 4.6. Nonequivariantly, $\lambda^{-1} \mathrm{MGLR} \simeq \mathrm{MGL}$.

Proof. Notice that nonequivariantly, $\lambda$ realizes as

$$
\begin{array}{r}
S^{2+2 \alpha} \simeq \Sigma \mathbb{G}_{m} \wedge \Sigma \mathbb{G}_{m} \rightarrow \Sigma \mathbb{G}_{m} \times \Sigma \mathbb{G}_{m} \rightarrow B\left(\mathbb{G}_{m} \times \mathbb{G}_{m}\right) \rightarrow B G L_{2} \rightarrow \operatorname{MGL}(1) \\
\rightarrow \Sigma^{2+2 \alpha} \mathrm{MGL}
\end{array}
$$

Notice that this map is clearly nonzero, and represents an element in $\pi_{\star}(\mathrm{MGL})$ of degree 0 . Notice that the only nonzero element in $\pi_{\star}(M G L)$ of degree 0 is the identity element. Therefore, $\lambda^{-1}$ MGLR is nonequivariantly equivalent to MGL. 
Theorem 4.7. Localizing at $p=2$, we have that

$$
\mathrm{MGL}=\bigvee_{m_{i}} \Sigma^{m_{i}(1+\alpha)} \mathrm{BPGL}
$$

for integers $m_{i}$. There exists a spectrum BPGLR such that

$$
\operatorname{MGLR}=\bigvee_{m_{i}} \Sigma^{m_{i}(1+\sigma \alpha)} \operatorname{BPGLR}
$$

Furthermore, $\Phi^{C_{2}}(\mathrm{BPGLR})=\theta^{-1} H \mathbb{Z} / 2$.

\subsection{Calculating the coefficients of $\theta^{-1} \lambda^{-1}$ MGLR}

Proposition 4.8. There exists an element of order $1-\alpha$ in the Borel cohomology and the Tate cohomology of $\lambda^{-1}$ MGLR. We will call this element $\theta$.

Proof. Using simplicial $E C_{2}$, we can set up a Borel Cohomology Spectral Sequence for $\lambda^{-1}$ MGLR as follows. First we note that since we have inverted $\lambda$, we can choose to ignore all $\sigma \alpha$ grading, and instead only consider the grading $*+*^{\prime} \sigma+*^{\prime \prime} \alpha$. Moreover, we will filter by $\alpha$ twists. In other words, we will consider the grading $*+*^{\prime} \sigma+k \alpha$ for fixed $k$. Now for each $k \leq 0$, we have a bijection between the motivic Borel Cohomology Spectral Sequence of $\lambda^{-1}$ MGLR and the classical Borel Cohomology Spectral Sequence of MR. This is true since $\lambda^{-1}$ MGLR is nonequivariantly MGL, and over $\mathbb{C}$, there is a bijection between $\pi_{*+k \alpha}(\mathrm{MGL})$ and $\pi_{*}(\mathrm{MU})$. It follows that the motivic Borel Cohomology Spectral Sequence associated to $\lambda^{-1} \mathrm{MGLR}_{*+*^{\prime} \sigma+*^{\prime \prime} \alpha}$, where $*, *^{\prime} \in \mathbb{Z}$ and $*^{\prime \prime} \in \mathbb{Z}^{\leq 0}$, converges to $\pi_{*+*^{\prime} \sigma+*^{\prime \prime} \alpha}\left(F\left(E C_{2+}, \lambda^{-1} \mathrm{MGLR}\right)\right) \cong \pi_{\star}(\mathrm{MR})[\theta]$. It follows that $\theta \in \lambda^{-1}$ MGLR. The same argument works for the Tate cohomology of $\lambda^{-1}$ MGLR.

Corollary 4.9. There exists an element of degree $1-\alpha$ in the coefficients of $\lambda^{-1}$ MGLR. We again call this element $\theta$. 
Proof. This follows by considering the following square originating from the Tate diagram,

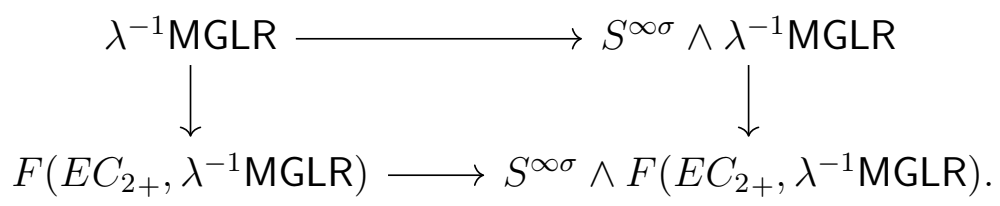

It is easy to see that the element $\theta \in \pi_{\star}\left(F\left(E C_{2+}, \lambda^{-1} \mathrm{MGLR}\right)\right)$ is sent to $\theta \in$ $\pi_{\star}\left(S^{\infty \sigma} \wedge F\left(E C_{2+}, \lambda^{-1} \mathrm{MGLR}\right)\right)$. This is true since the topological realization of $\theta$ is just 1, and since the Borel and Tate cohomology spectral sequences of $\lambda^{-1}$ MGLR and MR are isomorphisms for fixed alpha twist $k \alpha, k \leq 0$. Now, notice that there is an easily described twist in $\pi_{\star}\left(S^{\infty \sigma} \wedge \lambda^{-1} \mathrm{MGLR}\right)$ of degree $1-\alpha$, which we also call $\theta$. If $s$ is the euler class $s \in \pi_{-\sigma}(\mathrm{MGLR})$, and $t$ is the euler class $t \in \pi_{-\sigma \alpha}(\mathrm{MGLR})$, the $\theta \in \pi_{1-\alpha}\left(S^{\infty \sigma} \wedge \lambda^{-1} \mathrm{MGLR}\right)$ is given by $\lambda s^{-1} t$. By comparison with topology, and in view of the fact that the topological realization of $\theta$ is 1 , it follows that $\theta \in$ $\pi_{1-\alpha}\left(S^{\infty \sigma} \wedge \lambda^{-1} \mathrm{MGLR}\right)$ is sent to $\theta \in \pi_{1-\alpha}\left(S^{\infty \sigma} \wedge F\left(E C_{2+}, \lambda^{-1} \mathrm{MGLR}\right)\right.$. Therefore, the element named $\theta$ commutes in the bottom row and rightmost column of the diagram corollary 4.9. Since the commutative square corollary 4.9 is a pullback, there must exist an element $\theta \in \pi_{\star}\left(\lambda^{-1} \mathrm{MGLR}\right)$ which is sent to $\theta \in \pi_{\star}\left(F\left(E C_{2+}, \lambda^{-1} \mathrm{MGLR}\right)\right)$.

Now, as we inverted $\lambda \in \pi_{1-\sigma+\sigma \alpha-\alpha}(\mathrm{MGLR})$, so too can we invert $\theta \in \pi_{1-\alpha}(\mathrm{MGLR})$. This gives us a spectrum $\theta^{-1} \lambda^{-1}$ MGLR. In its coefficients, the element $\lambda^{-1} \theta$ has degree $\sigma-\sigma \alpha$ and is invertible.

Proposition 4.10. $\left(S^{\infty \sigma+\infty \sigma \alpha} \wedge \theta^{-1} \lambda^{-1} \mathrm{MGLR}\right)^{C_{2}} \simeq\left(S^{\infty \sigma} \wedge \theta^{-1} \lambda^{-1} \mathrm{MGLR}\right)^{C_{2}}$.

Proof. To simplify notation, we write

$$
E:=S^{\infty \sigma} \wedge \theta^{-1} \lambda^{-1} \mathrm{MGLR}, F:=S^{\infty \sigma+\infty \sigma \alpha} \wedge \theta^{-1} \lambda^{-1} \mathrm{MGLR} .
$$

Notice that $\Sigma^{\sigma \alpha-\sigma} E \simeq E$ since $\theta \lambda^{-1} \in \pi_{\sigma-\sigma \alpha}(E)$ is invertible. Also, it is clear that $\Sigma^{\sigma} E \simeq E$. Putting this together, we have that $\Sigma^{\sigma \alpha} E \simeq E$. Therefore, it follows that 
$F=\Sigma^{\infty \sigma \alpha} E \simeq E$.

Theorem 4.11. $\pi_{\star}\left(\theta^{-1} \mathrm{BPGLR}\right)=\pi_{\star}(\mathrm{BPR})\left[\lambda^{ \pm 1}, \theta^{ \pm 1}\right]$. Here, $\pi_{\star}(\mathrm{BPR})=$

$$
\begin{aligned}
& \mathbb{Z}_{(2)}\left[v_{n, l}, a \mid n \geq 0, l \in \mathbb{Z}\right] /\left(\begin{array}{c}
v_{0,0}=2, \\
a^{2^{n+1}-1} v_{n, l}=0, \\
\text { for } n \leq m: v_{m, k} \cdot v_{n, l 2^{m-n}}=v_{m, k+l} \cdot v_{n, 0}
\end{array}\right), \\
& |a|=-\sigma,\left|v_{n, l}\right|=\left(2^{n}-1\right)(1+\sigma)+l 2^{n+1}(\sigma-1)
\end{aligned}
$$

Proof. The claim is clear by comparison with topology [HK01]. In more detail, considering the commutative square of corollary 4.9 , the $C_{2}$ fixed points of the top right corner is easily seen to be equal to $\pi_{*}(\mathrm{MO})\left[\theta^{ \pm 1}\right]$. The bottom right corner is calculated by comparing the Tate cohomology spectral sequence for $\theta^{-1} \lambda^{-1}$ MGLR to topology. One deduces from the calculation that the $C_{2}$ fixed points of the the top and bottom right hand column are equal. From this it follows that $\theta^{-1} \lambda^{-1}$ MGLR is equal to its Borel cohomology. By comparing with topology, the claim follows. 


\section{BIBLIOGRAPHY}




\section{BIBLIOGRAPHY}

[ADF14] Aravind Asok, Brent Doran, and Jean Fasel. Smooth models of motivic spheres. arXiv preprint arXiv:1408.0413, 2014.

[Bac15] Tom Bachmann. The Hurewicz and Conservativity Theorems for $\mathbf{S H}(\mathbf{k})$ to $\mathbf{D M}(\mathrm{k})$. arXiv preprint arXiv:1506.07375, 2015.

[Bor03] Simone Borghesi. Algebraic Morava K-theories. Inventiones mathematicae, 151(2):381-413, 2003.

[DI05] Daniel Dugger and Daniel C. Isaksen. Motivic cell structures. Algebraic \& Geometric Topology, 5(2):615-652, 2005.

[EKMM07] Anthony D. Elmendorf, Igor Kriz, Michael A. Mandell, and J. Peter May. Rings, modules, and algebras in stable homotopy theory. Number 47. American Mathematical Society, 2007.

[GM95] John Patrick Campbell Greenlees and J. Peter May. Generalized Tate cohomology, volume 543. American Mathematical Society, 1995.

[HK01] Po Hu and Igor Kriz. Real-oriented homotopy theory and an analogue of the Adams-Novikov spectral sequence. Topology, 40(2):317-399, 2001.

[HK11] Po Hu and Igor Kriz. Topological Hermitian Cobordism. Journal of Homotopy and Related Structures, pages 1-25, 2011.

[HKO11] Po Hu, Igor Kriz, and Kyle Ormsby. The homotopy limit problem for Hermitian K-theory, equivariant motivic homotopy theory and motivic Real cobordism. Advances in Mathematics, 228(1):434-480, 2011.

[Hoy15] Marc Hoyois. From algebraic cobordism to motivic cohomology. Journal für die reine und angewandte Mathematik (Crelle's Journal), 2015(702):173-226, 2015.

[HVØ16] Jeremiah Heller, Mircea Voineagu, and Paul Arne Østvaer. Topological comparison theorems for Bredon motivic cohomology. arXiv preprint arXiv:1602.07500, 2016.

[Jar87] John Frederick Jardine. Simplical presheaves. Journal of Pure and Applied Algebra, 47(1):35-87, 1987. 
[Lan67] Peter S. Landweber. Fixed point free conjugations on complex manifolds. Annals of Mathematics, pages 491-502, 1967.

[Lan68] Peter S. Landweber. Conjugations on complex manifolds and equivariant homotopy of MU. Bulletin of the American Mathematical Society, 74(2):271-274, 1968.

[Mil58] John Milnor. The Steenrod algebra and its dual. Annals of Mathematics, pages 150-171, 1958.

[MS16] John Milnor and James D. Stasheff. Characteristic Classes. (AM-76), volume 76. Princeton University Press, 2016.

[MV99] Fabien Morel and Vladimir Voevodsky. $\mathbb{A}^{1}$-homotopy theory of schemes. Publications Mathématiques de l'IHÉS, 90(1):45-143, 1999.

[MVW11] Carlo Mazza, Vladimir Voevodsky, and Charles A. Weibel. Lecture notes on motivic cohomology, volume 2. American Mathematical Society, 2011.

[Pen82] David J. Pengelley. The mod two homology of MSO and MSU as $\mathcal{A}$ comodule algebras, and the cobordism ring. Journal of the London Mathematical Society, 2(3):467-472, 1982.

[Sta16] Sven-Torben Stahn. The motivic Adams-Novikov spectral sequence at odd primes over $\mathbb{C}$ and $\mathbb{R}$. arXiv preprint arXiv:1606.06085, 2016.

[SV14] Alexander Smirnov and Alexander Vishik. Subtle characteristic classes. arXiv preprint arXiv:1401.6661, 2014.

[Tot99] Burt Totaro. The Chow ring of a classifying space. In Proceedings of Symposia in Pure Mathematics, volume 67, pages 249-284. Providence, RI; American Mathematical Society; 1998, 1999.

[Voe03] Vladimir Voevodsky. Reduced power operations in motivic cohomology. Publications Mathématiques de l'IHÉS, 98:1-57, 2003. 